\title{
Numerical Analysis of a High-Order Unstructured Overset Grid Method for Compressible LES of Turbomachinery
}

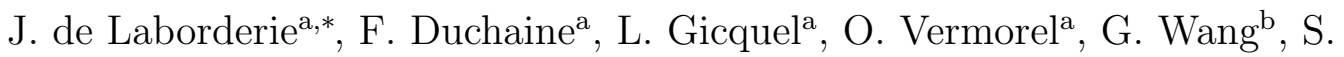 \\ Moreau ${ }^{\mathrm{b}}$ \\ ${ }^{a}$ CFD Team, CERFACS, 42 avenue Gaspard Coriolis, 31057 Toulouse, France \\ ${ }^{b}$ Department of Mechanical Engineering, University of Sherbrooke, Sherbrooke, J1K 2R1, \\ Qc, Canada
}

\begin{abstract}
Large-Eddy Simulation (LES) is recognized as a promising method for highfidelity flow predictions in turbomachinery applications. The presented approach consists of the coupling of several instances of the same LES unstructured solver through an overset grid method. A high-order interpolation, implemented within this coupling method, is introduced and evaluated on several test cases. It is shown to be third order accurate, to preserve the accuracy of various second and third order convective schemes and to ensure the continuity of diffusive fluxes and subgrid scale tensors even in detrimental interface configurations. In this analysis, three types of spurious waves generated at the interface are identified. They are significantly reduced by the high-order interpolation at the interface. The latter having the same cost as the original lower order method, the high-order overset grid method appears as a promising alternative to be used in all the applications.
\end{abstract}

Keywords: Large-Eddy Simulation, overset grids, high-order interpolation, turbomachinery.

\footnotetext{
*Corresponding author

Email addresses: jerome.de.laborderie@cerfacs.fr (J. de Laborderie), florent.duchaine@cerfacs.fr (F. Duchaine), laurent.gicquel@cerfacs.fr (L. Gicquel), olivier.vermorel@cerfacs.fr (O. Vermorel), gfwang@zju.edu.cn (G. Wang), stephane.moreau@usherbrooke.ca (S. Moreau)
} 


\section{Introduction}

In Computational Fluid Dynamics (CFD), the Large Eddy Simulation (LES) approach has been developed over many years to numerically compute turbulent flows [1-3]. This approach has always been seen as a trade-off between cost and accuracy, typically located between the strict resolution of all the space and time flow scales as performed in Direct Numerical Simulation, and the averaging of the turbulent flow structures as realized in Reynolds-Averaged Navier-Stokes (RANS) methods. Indeed the principle of LES consists in the resolution of the large scales of the flow and the modeling of the smallest unresolved scales. This modeling assumes that these smallest flow scales follow a universal dissipative behavior. The recent advancement in High-Performance Computing (HPC) coupled to a massive parallelization of flow solvers has allowed the application of LES to actual complex geometries. For instance, in the field of aeronautical propulsion, it is now commonly admitted that the LES technique is required to simulate reacting flows inside a combustion chamber with sufficient accuracy while keeping the cost affordable [4]. The application of LES to turbomachinery stages is less mature, due to its higher cost and the inherent complexity of the flow, driven by wall flow behaviors, high Mach and Reynolds numbers and domains in relative motions. Nevertheless recent studies tend to show the benefit of such simulations over existing RANS computations, still widely used in industry [5-8].

For the simulation of turbomachinery, two main approaches exist to transfer the flow through successive bladed rows in relative motion. These are namely the sliding interface method (e.g. [9-11]) and the overset grid method. The principle of the overset grid method consists in the discretization of the flow on different meshes, that are coupled over a common geometrical zone so as to allow the proper exchange of the necessary information. This method has been widely developed over several decades, starting with the early works of Schwarz [12] and Volkov [13], then followed by many authors, e.g. [14-24]. Overlapping grids have been used in several fields of application, e.g. in com-

putational aeroacoustics [25, 26], conjugate heat transfer [27], simulation of moving bodies for aeronautical [28] and marine purposes [29]. For turbomachinery simulations with a structured solver, the overset grid approach is a way to account for complex technological effects such as cavities and casing treatments $[30,31]$. With respect to other methods, the overset grid method is known to be more flexible since the size of the overlapping zone can vary 
and the mesh generation of complex geometries is eased [23]. However the main challenge of such a method whenever applied in the context of LES consists in minimizing dissipative and dispersive errors during the transfer of information between two overlapping domains. This is even more critical in compressible LES since the resolved vortical, acoustic and entropic waves must accurately cross the overlapping interface. That is the reason why highorder overset grid methods have been recently developed for structured grids, for steady or moving interfaces [25, 32-36]. High-order discontinuous numerical methods, such as Discontinuous Galerkin, Flux Reconstruction and Spectral methods, have recently emerged in the CFD community, and dedicated high-order overset grid techniques had to be developed to preserve accuracy properties of such methods, e.g. in [22-24, 37]. The development of high-order overset grid methods for unstructured grids has been less extensive whether for a steady interface $[38,39]$ or for a moving interface $[40,41]$. Recently, an overset methodology has been proposed to perform LES of turbomachinery stages. The approach relies on the coupling of several instances of the same high-order and unstructured LES solver with the so-called MISCOG technique: Multi-Instance Solver Coupled through Overlapping Grids [42].

MISCOG has already been applied to several types of turbomachinery stages [43-46] and the obtained results are very promising. Besides these preliminary validations and proofs of concepts, there is now the need to perform more fundamental analyses of MISCOG, in order to precisely evaluate the accuracy of the proposed high-order overset grid method for unstructured grids and moving interfaces. Indeed, while the original MISCOG method was introduced with a second order interpolation [42], a higher order interpolation scheme has since been added. The effects of non-coincident overlapping zones, moving grids, and interpolation schemes have still to be systematically studied in this new specific context. In parallel it should be stressed that such interface treatments are also to be considered as numerical discontinuities for the flow solver possibly generating undesired waves [47]. This point, hardly addressed within the context of an overset method in the literature, needs to be studied in-depth so as to fully apprehend available predictions and their quality. The present paper is split as follows: first, the LES solver and the principle of the overset grid method are recalled before describing the implementation of the high-order interpolation. Its accuracy is then precisely measured in the the third section. The fourth section is dedicated to an inviscid vortex test case defined to analyze the interaction between MISCOG and convective schemes. To evaluate the behavior of diffusive fluxes, a vis- 
cous vortex test case is presented in the fifth section. The 3-D flow around a cylinder is then chosen as the case to assess the interface treatment in the near wake of a solid body, since this configuration could be found in an actual turbomachinery stage. Finally the seventh section addresses the stability of MISCOG in studying a test case with a strong shock.

\section{LES solver and interface treatment}

\subsection{Numerical method for compressible flows}

The LES solver AVBP [48, 49], jointly developed by CERFACS and IFPEN, resolves 3-D compressible unsteady reacting flows on unstructured grids. The filtered Navier-Stokes equations describing conservation of mass, momentum and energy without chemical reaction can be written as:

$$
\frac{\partial \mathbf{W}}{\partial t}+\nabla \cdot \mathcal{F}=0
$$

W contains the conservative variables $(\rho, \rho \mathbf{U}, \rho E)$ where $\rho$ is the fluid density, $\mathbf{U}$ is the velocity vector and $E$ is the total energy of the fluid. $\mathcal{F}$ is the flux tensor, decomposed into the convective part: $\mathcal{F}^{c}(\mathbf{W})$ and the viscous part: $\mathcal{F}^{v}(\mathbf{W}, \nabla \mathbf{W})$. This viscous flux contains the contributions of the unresolved flow scales whenever derived in the context of LES. For non-reacting and single-species flows, these contributions are modeled under the form of the Sub-Grid Scale (SGS) stress tensor, using the Boussinesq assumption [1]:

$$
\tau_{i j}^{t}=2 \rho \nu_{t}\left(S_{i j}-\frac{1}{3} \delta_{i j} S_{l l}\right),
$$

where $S_{i j}$ is the resolved strain rate tensor and $\nu_{t}$ is the SGS turbulent viscosity to be modeled. Several SGS models are implemented in AVBP,

including the Wall Adapting Local Eddy-viscosity model (WALE) [50] for which the turbulent viscosity is written as:

$$
\nu_{t}=\left(C_{w} \Delta\right)^{2} \frac{\left(s_{i j}^{d} s_{i j}^{d}\right)^{3 / 2}}{\left(S_{i j} S_{i j}\right)^{5 / 2}+\left(s_{i j}^{d} s_{i j}^{d}\right)^{5 / 4}},
$$

with 


$$
\begin{aligned}
s_{i j}^{d} & =\frac{1}{2}\left(g_{i j}^{2}+g_{j i}^{2}\right)-\frac{1}{3} g_{k k}^{2} \delta_{i j}, \\
g_{i j} & =\frac{\partial u_{i}}{\partial x_{j}}
\end{aligned}
$$

and $u_{i}$ the $i^{t h}$ component of the resolved velocity, $\Delta$ the filter width and $C_{w}$ $=0.5$ the WALE coefficient.

In the AVBP solver, the discretization is performed using the cell-vertex method [51]. Conservation relations are thus applied to grid cells, and solutions are stored at grid vertices (or nodes) [52]. In this approach, the gradient of a scalar $A$ at a given cell $C$ is calculated using Green-Gauss theorem:

$$
(\vec{\nabla} A)_{C}=\frac{1}{V_{C}} \sum_{f \in C} A_{f} \overrightarrow{d S}_{f},
$$

where $V_{C}$ is the volume of the cell $C, f$ are the faces of $C$, with their oriented surfaces $S_{f}$, and $A_{f}$ are the average values of $A$ over each face. The nodal-based gradient at node $j$ is then approximated using a volumeweighted average of cell-based gradients from the cells $C_{i}$ adjacent to node $j$ :

$$
(\vec{\nabla} A)_{j} \approx \frac{1}{V_{j}} \sum_{i} V_{C_{i}}(\vec{\nabla} A)_{C_{i}}
$$

where $V_{j}$ is the volume of the dual cell associated with node $j$. This calculation of nodal gradients uses a compact stencil and is linearity preserving, thus second-order accurate $[53,54]$. No limiter is applied to nodal gradients, however a corrective term in the gradient expression has been added to damp oscillatory modes that may exist on boundaries $[54,55]$.

Among the numerical convective schemes implemented within AVBP, three of them are specifically employed in this paper since they are commonly recommended for turbomachinery applications. These schemes have already been extensively studied, so only their main characteristics are recalled. First the Lax-Wendroff (LW) scheme is second order accurate in space and time $[56,57]$. Second TTG4A is third order in space and fourth order in time $[58,59]$, and TTGC is third order in space and time [60]. For each of these three schemes, the time marching method is explicit and a full discretization allows replacing time derivatives by space derivatives [60]. The LW scheme 
thus contains a one step time marching method, and the high-order TTG schemes use a two-step approach. Even if LW is only second-order accurate, the interest of using a high-order interface treatment with this scheme will be demonstrated in Secs. 5.3 and 6 .

Using the von Neumann analysis for an advection equation, regularly discretized over a 1-D domain, the dissipative and dispersive properties of these schemes are recalled in Figs. 1(a) and 1(b) respectively, from [52]. Only the results for a CFL equals to 0.7 are shown since this value is used for the entire analysis in this paper. $k$ is the wavenumber and $\Delta x$ is the element size. It is shown that LW is more dissipative than TTG schemes for high frequency waves. TTG schemes have correct dispersive properties up to $k \Delta x \approx 2$ since the propagation velocity of the waves are weakly modified. For higher wavenumbers, TTGC decreases this velocity up to 0, meaning that the smallest waves do not propagate. In addition their amplitudes are not significantly reduced, making this scheme sensitive to Nyquist frequency waves. With TTG4A, high frequency waves are in advance of phase, and their amplitudes are moderately damped.

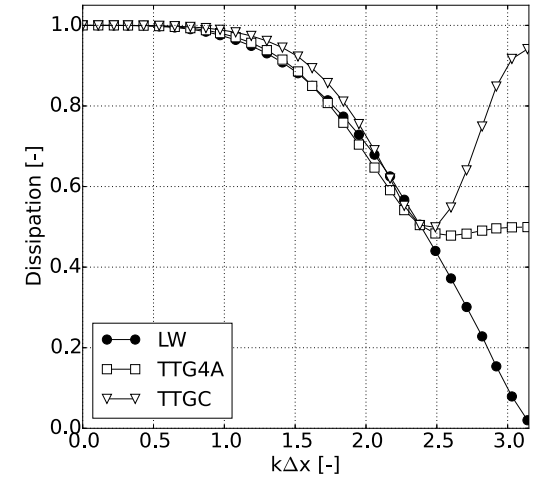

(a) Dissipation.

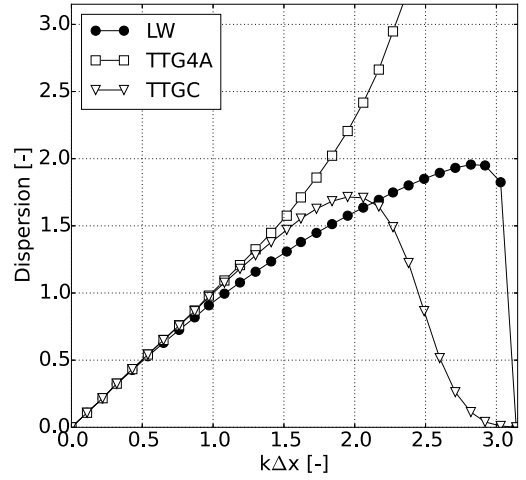

(b) Dispersion.

Figure 1: Dissipation and dispersion properties of the schemes LW, TTG4A and TTGC, for $\mathrm{CFL}=0.7$, as a function of the dimensionless wavenumber $k \Delta x$.

\subsection{Overset grid method}

The LES solver for turbomachinery flows TurboAVBP [42] is based on the coupling of several instances of the above described solver AVBP. Each 
computational domain with its own grid velocity is attributed to a dedicated instance. Typically, an instance is dedicated to fixed bladed rows (stators) and another instance is dedicated to moving bladed rows (rotors) (see e.g. [45]). In AVBP, movement and deformation of the mesh are performed with the ALE method [61]. For turbomachinery applications, only solid rotations are employed and the equations are solved in the absolute reference frame. The exchange of information between these instances is realized with the MISCOG overset grid method, introduced by Wang et al. [42].

The scope of MISCOG applications concerns turbomachinery flows. This overset grid method is intended to be used at each rotor-stator interface in a multi-row turbomachine. Its range of applications is therefore limited in comparison with other existing overset grid methods (e.g. [23, 24, 40, 41]), i.e. no hole cutting or arbitrary interface movement is targeted here. Indeed the displacement of moving domains are here restrained to solid rotation, possibly accelerated, around a fixed axis. The positions of interfaces, fixed in time, can be chosen in accordance with the mesh generation strategy. Typically it is recommended to use similar cell sizes in each grid within the overlapping zones. Donor cells and receptor points are described geometrically by parametric entities (planes, cones, discs, cylinders,...) depending on the treated configurations. The masking is fixed and no point is found as an orphan. The association between receptor points and donor cells is updated at each iteration due to relative motion of overlapping domains. In terms of exchange algorithms, a rotating interface is the same as a translating interface. This is why the conclusions of this paper, for which only test cases with translating interfaces are considered, apply directly to rotating interfaces.

The principle of MISCOG is here schematically explained. Consider Fig. 2 representing overlapping cells, respectively belonging to two different grids, the left $(\mathrm{LG})$ and the right $(\mathrm{RG})$ grids. The receptor node $A_{L}$ belongs to $\mathrm{LG}$ and the donor cell composed of nodes $A_{R}, B_{R}, C_{R}$ and $D_{R}$ belongs to RG. At each time step, Eq. (1) discretized with a given numerical scheme is applied to each grid LG and RG separately. Due to the cell-vertex formalism of the solver, this leads to the update of the conservative variables at each node of each grid. Then at the end of each time step, the conservative variables of the overlapping nodes are interpolated and exchanged between both grids. For example, conservative variables at node $A_{L}$ are replaced with the contribution of the four nodes $A_{R}, B_{R}, C_{R}$ and $D_{R}$. The interpolation required at this step is performed with Lagrange interpolators, expressed as: 


$$
\mathcal{L} f=\sum_{k=1}^{n} f\left(q_{k}\right) \phi_{k},
$$

where $f$ is a function approximated by Lagrange polynomial elements and $f\left(q_{k}\right)$ are the function values at the nodes $q_{k}$ of the element. $n$ is the number of nodes of the element and $\phi_{k}$ are its shape functions. The interpolation coefficients are computed using the local coordinates of the elements. In the original version of MISCOG [42], barycentric or bilinear interpolations are implemented, yielding an interpolation that is second order accurate.

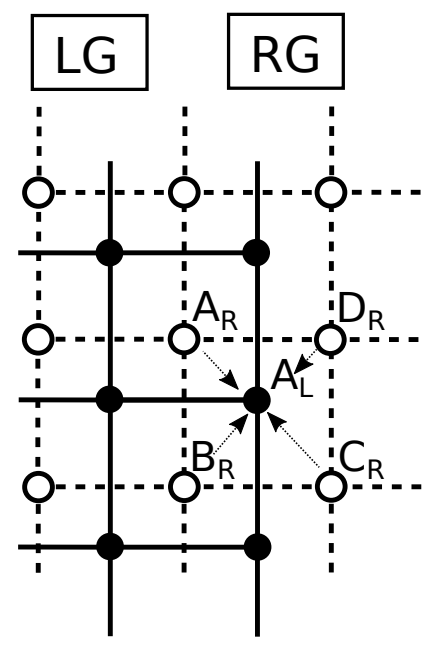

Figure 2: Sketch of overlapping cells in 2-D for two different grids LG (left grid, solid line) and RG (right grid, dashed line).

In practice, the coupling of several AVBP instances is externally performed with the parallel coupler OpenPALM, developed at CERFACS and ONERA [62]. OpenPALM is responsible for localization of receptor points in donor cells and for handling the communications between the processors on which the overlapping zones are distributed. The successive steps in the coupling procedure consist in finding the enclosed cell (defined by $\left(A_{R}, B_{R}\right.$, $C_{R}, D_{R}$ ) here), computing the local coordinates of $A_{L}$ in this cell, computing the interpolation coefficients using a shape function and computing the interpolated conservative variables at node $A_{L}$ using Eq. (6). The same methodology is simultaneously applied in the other direction, from the LG to the RG grids. The convergence and accuracy of this method depends on the 
amount of overlapping cells on each side of the interface. It has already been shown that this amount must be consistent with the stencil of the numerical scheme, and is generally found to increase with the order of the scheme [42]. The overset grid method without conservative interpolation is known to be not globally conservative, as recalled in $[24,29,37]$ for instance. This represents a limitation for its application to turbomachinery flows, for which mass flow conservation is expected. Note however that these conservation properties of MISCOG on mass flow and energy have already been addressed in [42]. To guarantee minimal losses on conservation, homogenous sizes of elements are recommended to be used. This point is further evaluated in Sec. 6.3.

\subsection{Extension to a high-order overset grid method}

MISCOG was originally proposed with a second order interpolation at the interface [42]. However the order of this interpolation should be at least equal to the order of the numerical scheme to preserve its accuracy [19]. That is the reason why a high-order interpolation has been implemented in MISCOG to comply with high-order schemes available in the LES solver. This implementation is based on the Hermite interpolator requiring first order derivatives of conservative variables that are already available at nodes within the solver, as described in Sec. 2.1. This approach is thus computationally efficient since it keeps the same stencil as the second order interpolation. This high-order interpolation has already been described analytically and used within CFD solvers, e.g. in [40]. However its implementation in a given code being not straightforward, this paper aims at demonstrating the expected performances of this high-order overset grid method within TurboAVBP. Only the main characteristics of the high-order interpolation are recalled here.

The interpolation is described for the particular case of a 3-D tetrahedral cell, schematically represented in Fig. 3. This cell has four nodes, numbered from $q_{1}$ to $q_{4}$, and 6 mid-edge nodes, numbered from $q_{5}$ to $q_{10}$. At point $P$, located inside this cell and belonging to an other grid, the conservative variables are interpolated according to the following successive steps.

First the barycentric coordinates of $P\left(\lambda_{1}, \lambda_{2}, \lambda_{3}, \lambda_{4}\right)$ inside the cell are computed, according to the general matrix form:

$$
\lambda=\mathbf{T}^{-1}\left(\mathbf{x}_{P}-\mathbf{x}_{q_{1}}\right),
$$

with 


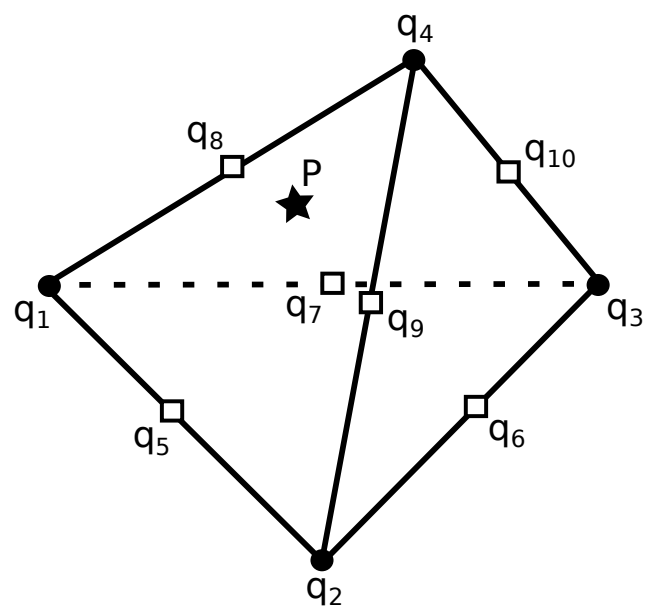

Figure 3: Sketch of a tetrahedral cell. $P$ is located inside this cell.

$$
\lambda=\left[\begin{array}{l}
\lambda_{2} \\
\lambda_{3} \\
\lambda_{4}
\end{array}\right]
$$

and $\mathbf{x}_{P}=\left(x_{1, P}, x_{2, P}, x_{3, P}\right)$ and $\mathbf{x}_{q_{1}}=\left(x_{1,1}, x_{2,1}, x_{3,1}\right)$ the Cartesian coordinates of $P$ and $q_{1}$ respectively. The transformation matrix $T$ is written as:

$$
\mathbf{T}=\left[\begin{array}{lll}
x_{1,2}-x_{1,1} & x_{1,3}-x_{1,1} & x_{1,4}-x_{1,1} \\
x_{2,2}-x_{2,1} & x_{2,3}-x_{2,1} & x_{2,4}-x_{2,1} \\
x_{3,2}-x_{3,1} & x_{3,3}-x_{3,1} & x_{3,4}-x_{3,1}
\end{array}\right] .
$$

By definition of barycentric coordinates, $\lambda_{1}$ is:

$$
\lambda_{1}=1-\lambda_{2}-\lambda_{3}-\lambda_{4} .
$$

Then the shape function for each node is expressed as: 


$$
\begin{aligned}
\phi_{i} & =\left(2 \lambda_{i}-1\right) \lambda_{i}, \quad i=[1,4] \\
\phi_{5} & =4 \lambda_{1} \lambda_{2} \\
\phi_{6} & =4 \lambda_{2} \lambda_{3} \\
\phi_{7} & =4 \lambda_{3} \lambda_{1} \\
\phi_{8} & =4 \lambda_{1} \lambda_{4} \\
\phi_{9} & =4 \lambda_{2} \lambda_{4} \\
\phi_{10} & =4 \lambda_{3} \lambda_{4}
\end{aligned}
$$

The conservative variables $f$ are then interpolated at all mid-edge nodes $q_{5}$ to $q_{10}$, using the 1-D Hermite formulation. For instance, for the edge whose nodes are $q_{1}$ and $q_{2}$, the interpolated value of $f$ at $q_{5}$ is:

$$
f\left(q_{5}\right)=\frac{1}{2}\left(f\left(q_{1}\right)+f\left(q_{2}\right)\right)+\frac{1}{8} \sum_{i=1}^{3}\left(x_{i, 1}-x_{i, 2}\right)\left(\frac{\partial f}{\partial x_{i}}\left(q_{1}\right)-\frac{\partial f}{\partial x_{i}}\left(q_{2}\right)\right),
$$

where the derivatives $\partial f / \partial x_{i}$ correspond to the nodal gradients of conservative variables, evaluated according to the method described in Sec. 2.1. Finally, the interpolated value at point $P$ is:

$$
f(P)=\sum_{k=1}^{10} \phi_{k} f\left(q_{k}\right) .
$$

It can be shown that the 1-D Hermite interpolator of Eq. (11) is fourth order accurate [40]. However for cost and numerical reasons, the final expression of the 3-D interpolator in Eq. (12) is rather composed of a linear combination of 1-D Hermite interpolations. This approximation deteriorates the order of accuracy of this 3-D interpolation. The latter relies on nodal gradients of the solver, that are second-order accurate (see Sec. 2.1). The actual accuracy of the interpolation will be measured in Sec. 3. For other types of elements, the principle of this high-order interpolation is similar to the expressions presented for a tetrahedral cell. Only the barycentric coordinates and the shape functions vary depending on the type of element. In addition to accuracy, the high-order interpolation has also to be assessed in terms of stability. This point is specifically addressed in Sec. 7 . 


\section{Spatial accuracy of the high-order overset grid method}

The high-order overset grid method introduced in Sec. 2.3 and implemented within TurboAVBP is here precisely evaluated in terms of spatial accuracy, for different types of elements. To this end, the widely used test case of the inviscid vortex [63] is chosen.

\subsection{Configurations and initial vortex}

In order to measure the accuracy of the interpolation within MISCOG, an isentropic vortex is imposed on a domain, called AVBP01, and interpolated onto a second domain AVBP02, as represented in Fig. 4. Both domains overlap each other, and their respective cells are not coincident. This vortex has a strength $\Gamma$ and a radius $R_{c}$, centered at $\left(x_{c}, y_{c}\right)$. The velocity and temperature perturbations induced by this vortex are:

$$
\begin{aligned}
& \tilde{V}_{x}(x, y)=-\Gamma \frac{y-y_{c}}{R_{c}} e^{-r^{2} / 2} \\
& \tilde{V}_{y}(x, y)=\Gamma \frac{x-x_{c}}{R_{c}} e^{-r^{2} / 2} \\
& \tilde{T}(x, y)=\frac{1}{2 C_{p}}(\Gamma)^{2} e^{-r^{2}}
\end{aligned}
$$

with $r=\sqrt{\left(x-x_{c}\right)^{2}+\left(y-y_{c}\right)^{2}} / R_{c}$ and $C_{p}=\gamma /(\gamma-1) R_{\text {gas }} . \gamma$ is the ratio of specific heats, $R_{\text {gas }}$ is the gas constant and $C_{p}$ is the heat capacity at constant pressure. The initial uniform velocity is zero, and only one iteration is performed using the time step of $10^{-15} \mathrm{~s}$, such that convective schemes do

not interfere with the evaluation of the interpolation. The vortex being solution of an isentropic flow, the initial flow is:

$$
\begin{aligned}
V_{x}(x, y) & =\tilde{V}_{x}(x, y) \\
V_{y}(x, y) & =\tilde{V}_{y}(x, y) \\
T(x, y) & =T_{0}-\tilde{T}(x, y) \\
P(x, y) & =P_{0}\left(\frac{T(x, y)}{T_{0}}\right)^{\frac{\gamma}{\gamma-1}} .
\end{aligned}
$$




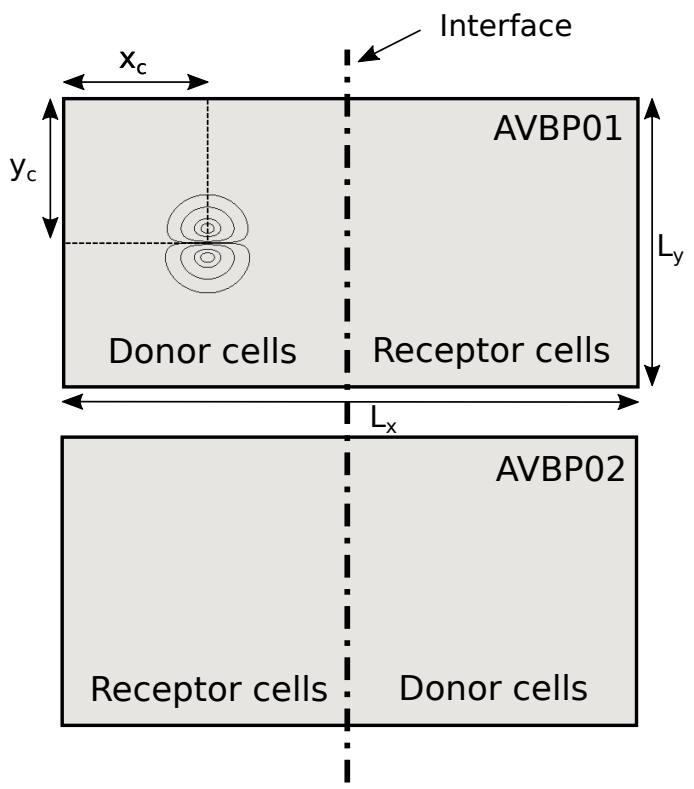

Figure 4: Domains AVBP01 and AVBP02, shifted vertically for visualization, at initial instant. Both domains overlap entirely. Vortex is imposed only in AVBP01.

The characteristic dimensions of the domains are $L_{x}=0.2 \mathrm{~m}, L_{y}=0.1$ $\mathrm{m}$, the vortex strength is set at $\Gamma=34.7 \mathrm{~m} / \mathrm{s}$ and its radius is $R_{c}=L_{y} / 20$. The background static temperature and pressure are respectively $T_{0}=300$ $\mathrm{K}$ and $P_{0}=1.10^{5} \mathrm{~Pa}$. This induces a pressure perturbation of the order of $700 \mathrm{~Pa}$. In AVBP01, all the cells at the left of the interface are donor, and the ones at the right of the interface are receptor. AVBP02 is in the reverse configuration. Thus MISCOG interpolates only once the vortex from AVBP01 to AVBP02. The error performed during this interpolation is then computed in AVBP02 only, comparing the interpolated vortex to the exact solution of Eq. (14). Noting $P_{i, \text { exact }}$ the associated analytical pressure field at node $i$, the mean quadratic error of the interpolated pressure $P_{i}$ is computed over the $\mathrm{N}$ nodes of the AVBP02 grid as:

$$
L_{2}(P)=\left[\frac{\sum_{i=0}^{N} V_{i}\left(P_{i, \text { exact }}-P_{i}\right)^{2}}{\sum_{i=0}^{N} V_{i}}\right]^{0.5}
$$




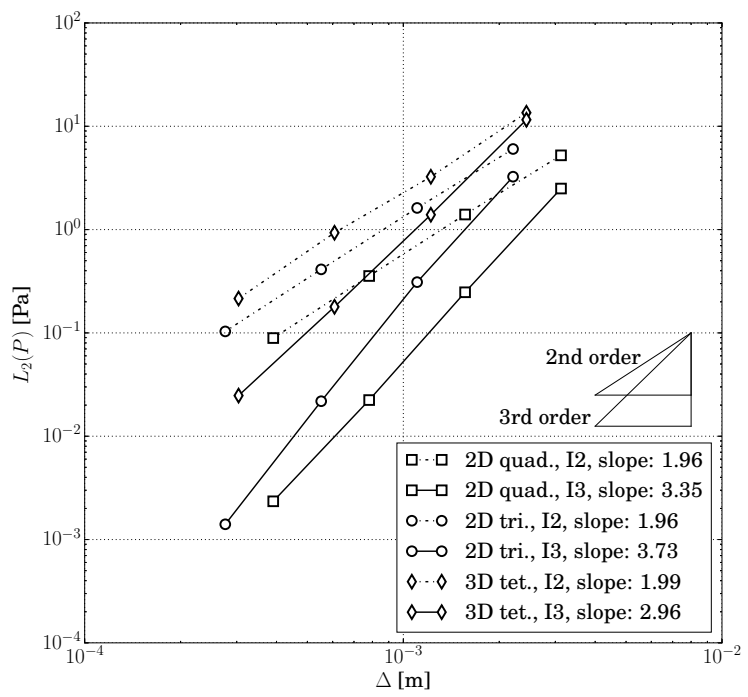

Figure 5: Quadratic error on the pressure, after interpolation from AVBP01 to AVBP02, for different element types: 2D quads, 2D triangles (tri.) and 3D tetrahedra (tet.).

where $V_{i}$ is the volume of the dual cell associated to node $i$.

\subsection{Results for 2-D and 3-D elements}

In order to measure the spatial accuracy of the MISCOG interpolation, several grids of successive refinements are built on the domains shown in Fig. 4. Each grid is composed of homogeneous elements, having the same size. Tests are performed for 2-D quads, 2-D triangles and 3-D tetrahedra cells. 3-D domains have around 10 cells in the z-direction. Cells are fully not coincident between both domains. For quads, AVBP02 nodes lie in the middle of AVBP01 cells. For triangles and tetrahedra cells, this repartition is random. For comparison purposes, an equivalent cell size $\Delta$ is defined as:

$$
\Delta=V^{1 / d},
$$

where $V$ is the volume of the cell and $d$ the dimension of the problem (2 or 3). The $L_{2}(P)$ errors computed over AVBP02, according to Eq. (15), as a function of the cell sizes $\Delta$, are shown in Fig. 5 .

The basic interpolation method in MISCOG, second-order accurate, is referred to as I2, whereas the high-order interpolation is denoted I3. What- 
ever the element type and the dimension of the problem, Fig. 5 confirms that the 12 treatment at the interface has a second-order spatial accuracy. It is further demonstrated that the high-order interpolation is third-order accurate in space for 3-D tetrahedra cells. This order is found to be between 3 and 4 for 2-D cells. Since the high-order interpolation is at least third-order accurate on the tested elements, this implies that nodal gradients computed in AVBP are indeed second-order accurate for these elements, as discussed in Sec. 2.1. These results are obtained for isotropic grids, that have already been recommended to be used in overlapping regions.

\section{Analysis of the overset grid method for a convected two-dimensional inviscid vortex}

Having presented the high-order interpolation and validating its spatial accuracy, the following sections aim at analyzing in depth the influence of the overset grid method on flows of increasing complexity. Indeed in LES, it is of primary importance to ensure that the interface treatment between two overlapping domains introduces minimal errors on every type of wave crossing this interface. To start, the test case of a convected inviscid vortex [63] is chosen in this section to evaluate the interaction of the interface treatments of MISCOG with AVBP convective schemes.

\subsection{Configurations}

The flow is considered inviscid and the 2-D Euler equations are solved. At time $t=0$, the initial solution corresponds to the superimposition of a uniform axial mean flow of velocity $U_{0}=c_{0} M$ with the isentropic vortex introduced in Sec. 3. $c_{0}$ is the speed of sound and $M$ is the Mach number. The initial solution corresponds to Eq. (14), except for the axial velocity becoming:

$$
V_{x}(x, y)=U_{0}+\tilde{V}_{x}(x, y) .
$$

This vortex is convected with the mean flow along the positive x-direction. It reaches its final position $x_{f}=x_{c}+L$ at time $t=T$. Periodic boundary conditions are imposed on the transversal edges of the domain, shown in Fig. 6. Characteristic inlet and outlet boundary conditions are imposed on the inlet (left side) and outlet (right side) edges, so that no spurious waves are reflected back into the domain $[64,65]$. Figure 6(a) corresponds to the reference case and is composed of a single domain (no interface). Figure 6(b) represents the coupled case in which the interface (dash line) lies between 
both domains respectively denoted AVBP01 and AVBP02. As indicated in Fig. 6(b), the overlapping region is composed of four cells on each side of the interface. In the particular case of Fig. 6(b), the overlapping cells are spatially coincident. All the grids are meshed with 2-D quads. The characteristic dimensions of the domains are $L_{x}=L_{y}=0.1 \mathrm{~m}$, the Mach number is set to $M=0.4$, and $T_{0}$ and $P_{0}$ are the same as in Sec. 3 .
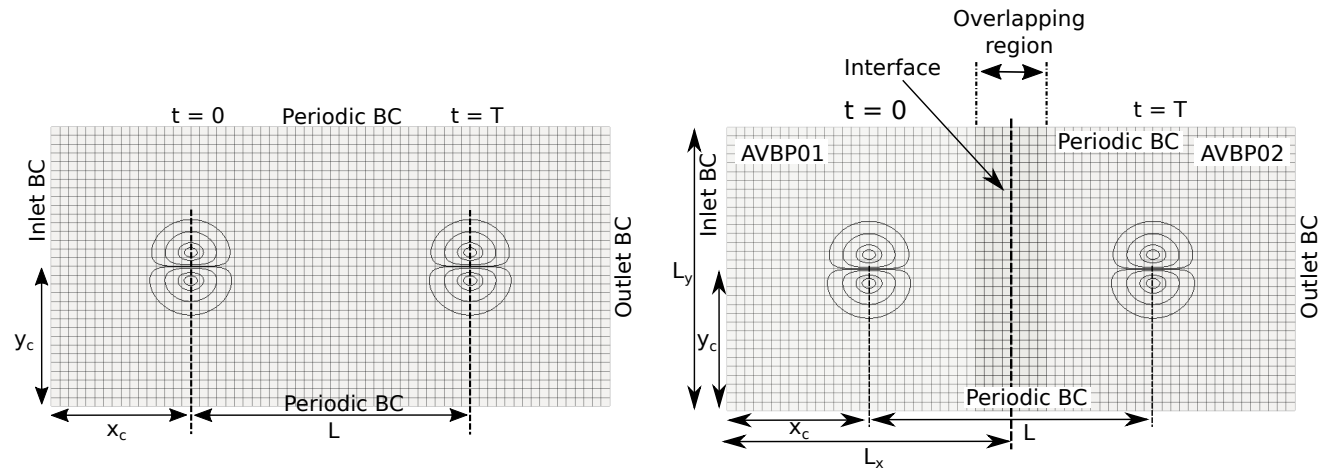

(a) Reference case, single grid, no interface (b) Coupled case, 2 grids, matching inter(REF case). face (QSM case).

Figure 6: Computational domains for the convected vortex test case.

One of the objectives consists in evaluating the properties of MISCOG for different interface configurations that may exist in actual applications. A set of cases is thus defined using the following nomenclature: QS means structured quads, M and NM mean matching and non-matching interfaces respectively, XY indicates the direction along which AVBP02 domain is shifted, and DXY the direction along which the cells of AVBP02 are coarsened. Figure 7(a) represents a zoom around the interface for the QSNMXY case. For the latter, the sizes of the elements are identical in both directions and in both domains $\left(\Delta x_{01}=\Delta y_{01}=\Delta x_{02}=\Delta y_{02}\right)$. The AVBP02 domain is shifted from $d_{y}$ and $d_{x}$ along $y$ or $x$ respectively, relative to the AVBP01 domain. To consider the most detrimental configuration, $d_{x}$ and $d_{y}$ are fixed such that:

$$
d_{x}=\Delta x_{01} / 2, d_{y}=\Delta y_{01} / 2
$$

Because of the necessary flexibility for unstructured mesh generation in real applications, element sizes may vary in both domains around the interface. Figure 7(b) represents the QSDXY case. The coarsening is chosen such 
that $\Delta y_{02}=2 \Delta y_{01}$ and $\Delta x_{02}=2 \Delta x_{01}$, thus corresponding to a drastic grid variation. Note that for this case, AVBP02 domain is also shifted from $d_{y}$ and $d_{x}$ to avoid coincident nodes in the coarsening direction.

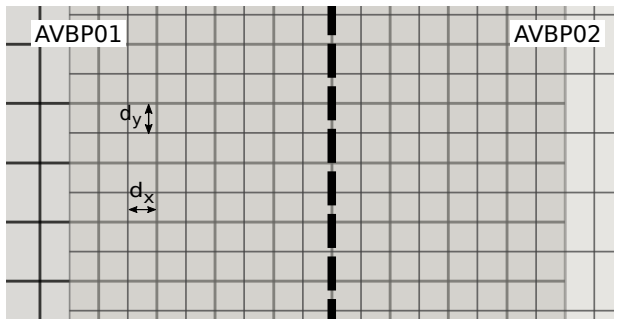

(a) QSNMXY case.

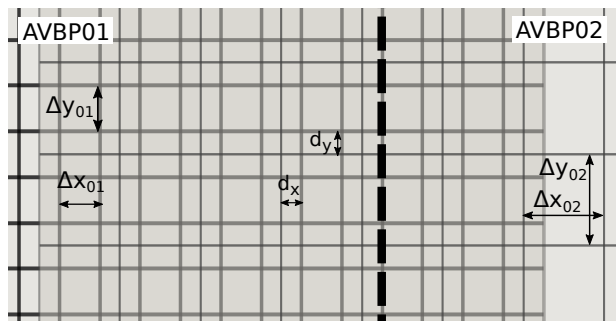

(b) QSDXY case.

Figure 7: Zoom on the tested interface configurations described in Tab. 1. Thick black lines belong to AVBP01 domain, thin gray lines belong to AVBP02 domain. The black dash line corresponds to the interface between both domains.

Table 1 gathers the interface configurations considered. In addition to these static simulations, the AVBP02 domain can also be translated along the y-direction, with a velocity $U_{\text {grid }}$. Here $U_{\text {grid }}$ is set to 0 or $300 \mathrm{~m} / \mathrm{s}$, this value being included in the nomenclature thereafter ( -0 or -300 respectively). These tests allow assessing the relative effect of a non-matching interface, a coarsened interface and a moving interface, as a function of the accuracy of the interpolation scheme at the interface, I2 or I3. As explained in Sec. 2.1, the convective schemes LW, TTG4A and TTGC, using no artificial viscosity, are successively tested with the constant value of 0.7 for the acoustic CFL number, defined as:

$$
C F L=\frac{\Delta t\left(U_{0}+U_{\text {grid }}+c_{0}\right)}{\Delta x} .
$$

For the second order LW scheme, no significant difference has been found when this scheme is applied to the cases of Tab. 1 together with the I2 or the I3 treatment at the interface. Thus only results obtained with high-order TTG schemes are presented in the rest of this section.

\subsection{Global evaluation}

The flow being inviscid, the exact solution of this problem is known to be Eqs. (14) and (17) in which $x_{c}$ is replaced with the actual axial location of the vortex at time $t$ following $x=U_{0} t$. Hence errors introduced by numerical 


\begin{tabular}{lll}
\hline \hline Configuration & Shift between AVBP01 & AVBP02 coarsening \\
name & and AVBP02 & \\
\hline \hline QSM & no & no \\
QSNMXY & $d_{x}, d_{y}$ & no \\
QSDXY & $d_{x}, d_{y}$ & $\Delta x_{02}=2 \Delta x_{01}, \Delta y_{02}=2 \Delta y_{01}$ \\
\hline \hline
\end{tabular}

Table 1: Interface configurations for the 2D inviscid vortex case.

schemes and interface treatments can be computed, and schemes orders can be measured. The mean quadratic error on the pressure is computed over each domain following Eq. (15). To assess the spatial order of the simulations, several grids of successive refinements are built, their number of nodes varying from $N_{x, 01}=N_{y, 01}=32$ to 256 along $L_{x}$ and $L_{y}$ respectively. Similarly to Sec. 3, the equivalent cell size $\Delta$ (Eq. (16)) is used to measure the error.

Figures 8 and 9 represent $L_{2}(P)$ errors at $t=T$ when the vortex has reached its final position, i.e. the center of AVBP02 domain, for configurations of Table 1. The averaged slopes of these errors are indicated on the figures. In Fig. 8, the curves termed REF (squares) represent the errors computed on the single domain (Fig. 6(a)). These are only associated to the error of the convective schemes and confirm that TTG4A and TTGC are at least third order accurate in space. In Fig. 8, the errors of the coincident coupled case (QSM, black crosses) are identical to those of the reference cases, independently of the interpolation at the interface (except for TTGC with smallest cell size, for which the small additional error originates from postprocessing method counting twice the error of overlapping zones). This result confirms that no interpolation takes place for a fully coincident overlapping zone thus the interface has no influence on the convection scheme.

In Fig. 8, curves with circles indicate fixed coupled cases, whereas diamonds refer to cases where the AVBP02 domain is translating at $U_{\text {grid }}=$ $300 \mathrm{~m} / \mathrm{s}$. For the high-order schemes tested, only the I3 interpolation allows preserving the order of the convective scheme. With a second order interpolation at the interface, the simulations degenerate to second order-type spatially accurate simulations, as already shown in [42]. With a static interface, the high-order interpolation gives results almost identical to cases with a matching interface (QSM). For TTGC, a moving interface tends to increase the global error relative to a static overlapping zone. This is caused by the 
spatial interpolation evolving in time due to the displacement of the AVBP02 cells. This point will be further explained and illustrated in Fig. 14. However this additional error due to the interface displacement does not deteriorate the schemes orders.

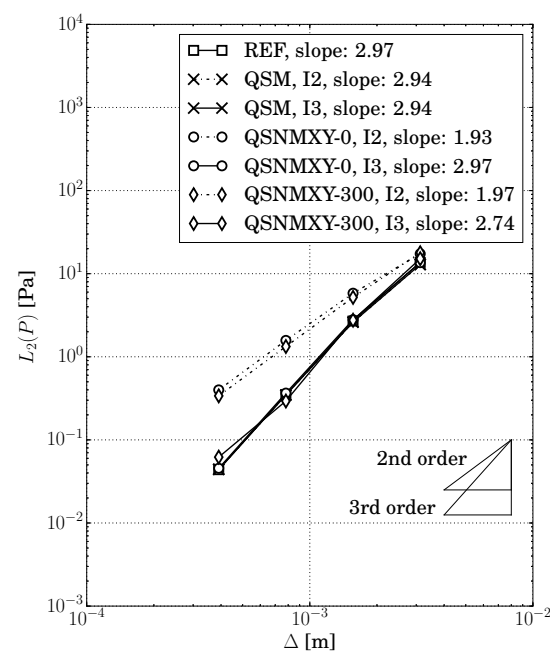

(a) TTG4A scheme.

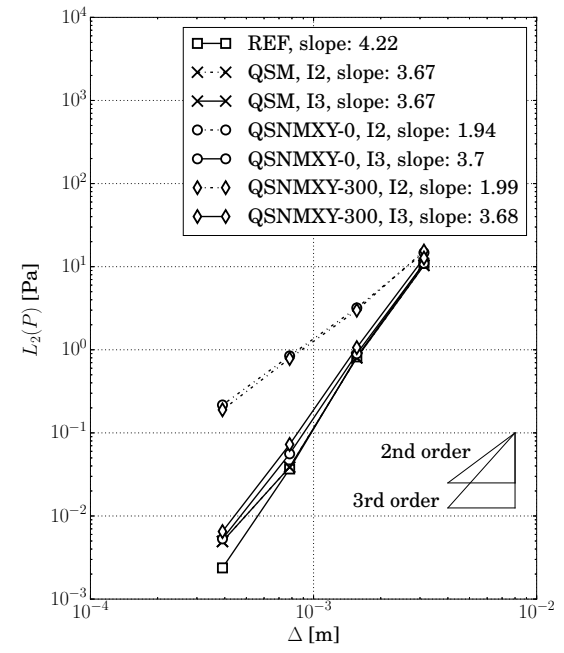

(b) TTGC scheme.

Figure 8: Quadratic error on the pressure, at time $t=T$, for a non-matching interface along the $\mathrm{x}$ and $\mathrm{y}$-directions (QSNMXY case).

Figure 9 corresponds to the case with a sudden variation of the element size at the interface. No reference case can be defined here since both effects of the interface and the grid coarsening occur. It is shown that, even if the orders of accuracy of TTG schemes are slightly lower than with uniform cell sizes, the high order interpolation improves this order by a value comprised between 0.7 and 1 in comparison with the basic I2 interface. The values of the quadratic errors are between one half and one order of magnitude higher than for the cases with uniform cells (QSNMXY). The additional error due to the displacement of the interface is higher when the cells dimensions vary in each overlapping zone.

In addition to the assessment of the global error at the last instant of simulations, it is relevant to evaluate its behavior during the convection of the vortex. Figures 10 and 11 show the temporal evolution of the quadratic error $L_{2}(P)$ from $t=0$ to $T$, for cases QSNMXY and QSDXY. Only the 


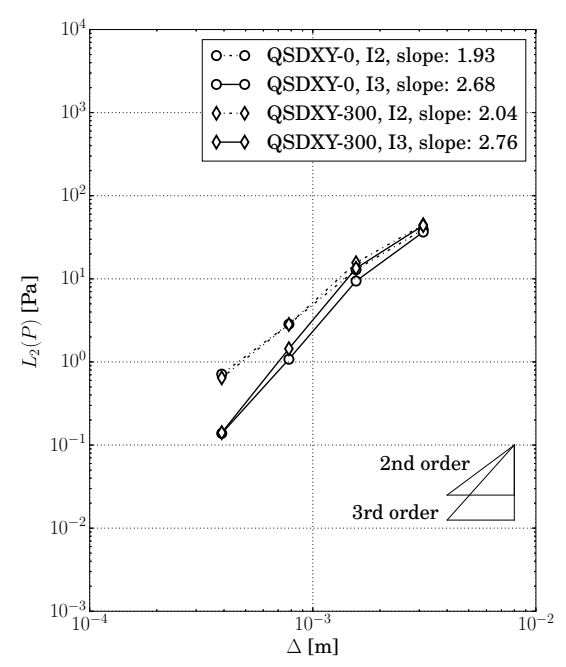

(a) TTG4A scheme.

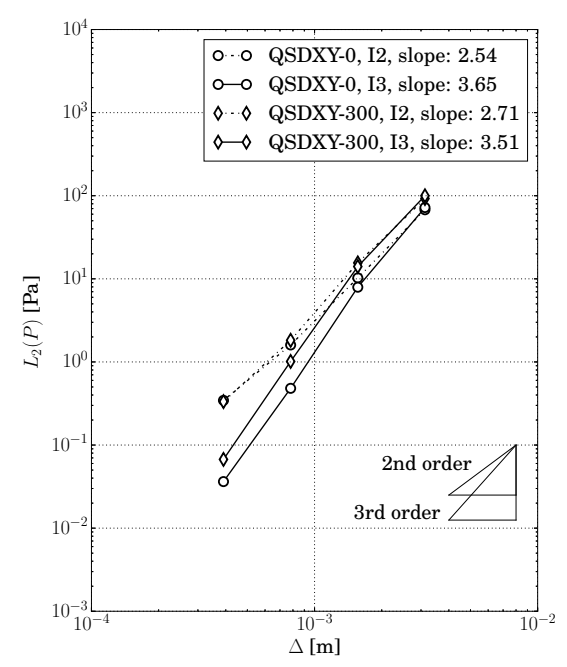

(b) TTGC scheme.

Figure 9: Quadratic error on the pressure, at time $t=T$, for AVBP02 grid coarsened along the $\mathrm{x}$ and $\mathrm{y}$-directions (QSDXY case).

most refined grids are considered. The reference curves correspond here to the case QSM of Fig. 10 (black crosses). For each scheme, the monotonic slope of the reference curve indicates the dissipative and dispersive errors of the convective scheme (see Sec. 2.1). It is first remarkable to notice that for a steady non-matching interface with a third order interpolation, the temporal evolution of quadratic error is almost identical to the reference, and not only at the last iteration as shown previously (white circles and plain line in Fig. 10). With a second order interpolation at the interface, the error sharply increases when the vortex crosses the interface (around $t / T=0.5$ ). As shown hereafter, this behavior is caused by the generation of spurious waves at the crossing of the interface by the vortex. This additional error then decreases to a steady value, due to the dissipation of these spurious waves by the scheme. For a moving interface with a I3 treatment, the error slightly increases once the vortex has crossed the interface and then tends to the reference value at the final time. Similar observations can be performed when the cell dimensions increase at the interface (Fig. 11), noting that the moving interface is responsible for larger errors compared to the fixed case, even for an I3 treatment at the interface. 


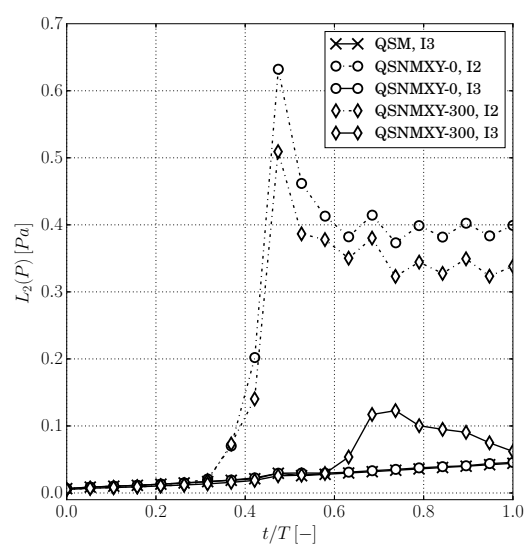

(a) TTG4A scheme.

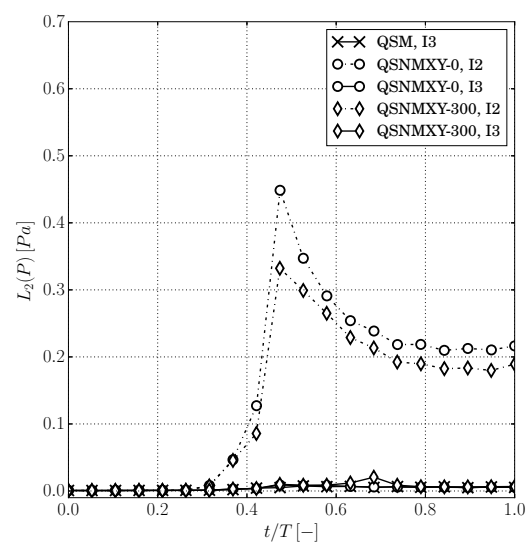

(b) TTGC scheme.

Figure 10: Temporal evolution of the quadratic error on the pressure for non-matching interface along x and y-directions (QSNMXY, $\left.N_{x, 01}=256\right)$.

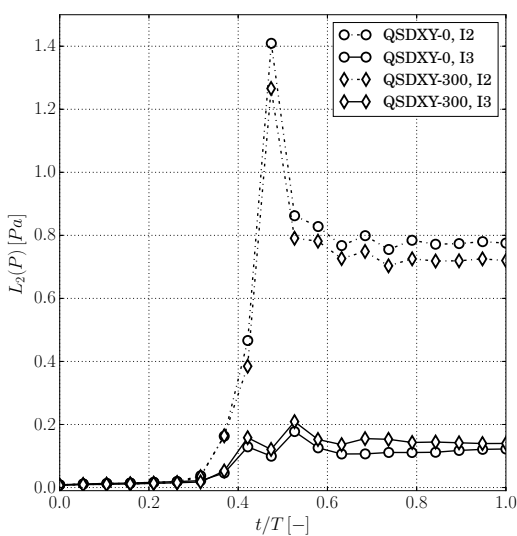

(a) TTG4A scheme.

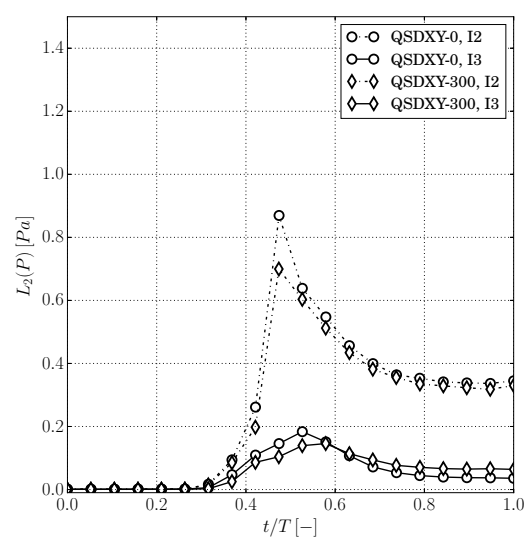

(b) TTGC scheme.

Figure 11: Temporal evolution of the quadratic error on the pressure for AVBP02 coarsening along x and y-directions (QSDXY, $N_{x, 01}=256$ ). 


\subsection{Detailed evaluation}

Having assessed the numerical treatment of the interface in terms of global error, the present section focuses on the local influence of MISCOG on the flow field. For a flow crossing the overlapping zones, the interface is seen as a numerical discontinuity, as would be a boundary condition or a solid surface. The effect of a discontinuity on numerical schemes has already been studied, for instance by Vichnevetsky and Bowles [47]. For a 1-D advection equation discretized by a centered scheme, these authors have shown that physical and numerical waves, respectively called $p$ and $q$ waves, are valid solutions of the problems. At a numerical discontinuity, both types of waves can be generated or transformed from one type to another depending on the treatment. The numerical waves are typically called Nyquist frequency waves and their wavelength of $2 \Delta x$ is the smallest one that can exist in the computational domain.

For the slightly more complex case of the convected vortex, where the Euler equations are discretized in 2-D, Fig. 12 represents the difference between the simulated pressure and the pressure computed in the reference case (REF, Fig. 6(a)), at $y=y_{c}$ and at several instants between $t=0$ and $t=T$. The observed signal hence contains only the effect of the interface and not the error associated with the convective scheme. Only the QSNMXY case with most refined grid and TTGC scheme is represented. It has been verified that similar features are present for LW and TTGA schemes. Figure 12(a) corresponds to a steady interface and Fig. 12(b) corresponds to the moving AVBP02 domain. The interface located at $x=0.1 \mathrm{~m}$ is indicated with a vertical dash line. The gray lines correspond to the 12 treatment at the interface while the black lines refer to the I3 treatment. For each case, when the vortex approaches the interface (around $0.4 \mathrm{~T}$ ), a small error appears. This dissipative and dispersive error, caused by the interface treatment on the pressure peak of the vortex, then amplifies and reaches its final position at $t=T$. Note that other phenomena are observed as the vortex crosses the interface. First, acoustic waves (pressure and velocity perturbations) are generated, traveling in the domain with a speed of $U_{0}-c_{0}$ (upstream propagation) or $U_{0}+c_{0}$ (downstream propagation). They are indicated with mixed dash lines. Additionally to theses physical acoustic waves generated, waves with much smaller wavelengths $(2 \Delta x)$ are clearly visible for the TTGC scheme (Figs. 12(a) and 12(b)), illustrating that this scheme is sensitive to Nyquist frequency waves as noted in Sec. 2.1. These $q$ waves, introduced in [47], are generated when the vortex crosses the interface. In addition to these 

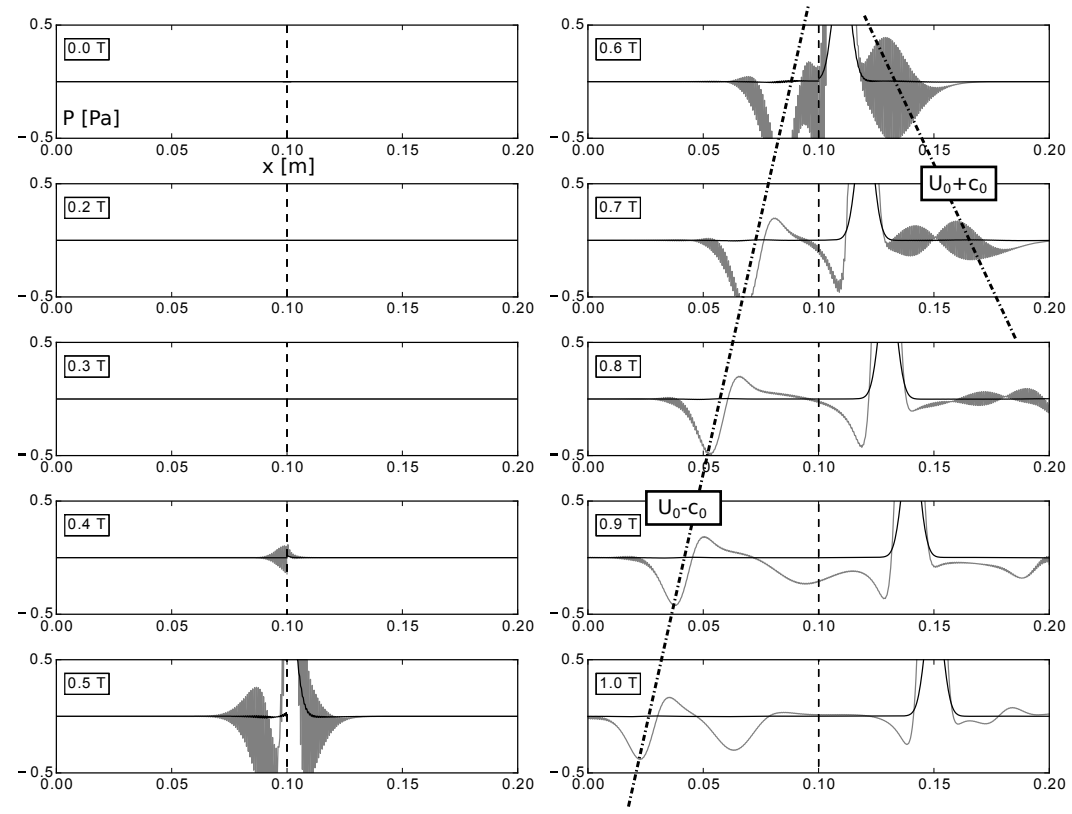

(a) $U_{\text {grid }}=0 \mathrm{~m} / \mathrm{s}$.
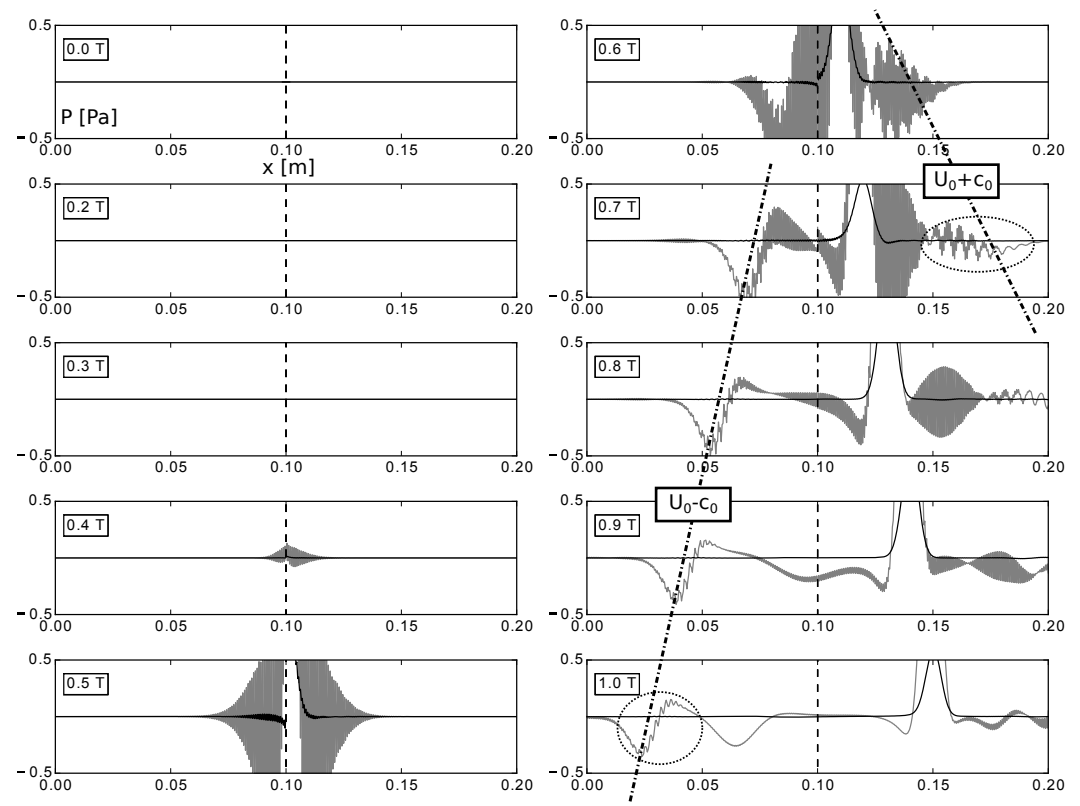

(b) $U_{\text {grid }}=300 \mathrm{~m} / \mathrm{s}$.

Figure 12: Pressure profiles, at $y=y_{c}$, at different instants between $t=0$ and $t=T$, relative to the reference case (REF). QSNMXY case, $N_{x, 01}=256$, TTGC scheme. Gray lines: second order interpolation at the interface. Black lines: third order interpolation at the interface. 
two trains of waves, other waves appear with a moving interface. They are evidenced in Fig. 12(b) (circles). These can be linked to the interpolation error that is unsteady when both domains are in relative motion (see comment about Fig. 14). Third order interpolation at the interface allows decreasing the amplitude of all these waves, with at least one order of magnitude gain if compared to the second order interpolation. It can also be observed that, once these waves are generated, their amplitudes rapidly decrease when they travel away from the interface thanks to the dissipative properties of the various schemes. All these features in part explain the reduction of the quadratic error observed after the interface crossing in Figs. 10 and 11.

While Fig. 12 allows a 1-D visualization of the problem, the latter is actually 2-D and the resulting pattern is more complex, since the generated waves may have different propagation directions depending on the characteristics of the $2 \mathrm{D}$ grids and schemes. This specificity is illustrated in Fig. 13 representing the pressure field at the instant when the vortex crosses the interface, for cases with the most refined grid and the TTGC scheme. With a second order interface (Fig. 13(a)), the three types of waves detailed above are clearly observed: acoustic waves, Nyquist frequency waves and waves issued by the moving interface (the latter are particularly visible in the AVBP02 domain). As expected, the third order interpolation at the interface significantly reduces the presence of all these spurious waves (Fig. 13(b)), and only the Nyquist frequency waves slightly appear.

To further assess the effect of a moving interface respectively to a fixed interface, a probe is located on the interface at $y=y_{c}$. Figure 14(a) shows the Fast Fourier Transform (FFT) spectrum of the pressure recorded in time at this location, for the QSNMXY case with the most refined grid $\left(N_{x}=256\right)$. The dashed lines correspond to the fixed interface and the plain lines indicate a moving interface with $U_{\text {grid }}=300 \mathrm{~m} / \mathrm{s}$. When the AVBP02 grid is moving, a clear spurious tone appears in the spectrum, at the frequency $F_{\text {grid }}=$ $U_{\text {grid }} / \Delta y$. As previously encountered, this spurious phenomenon is caused by the spatial interpolation error that is unsteady and periodic in time. Indeed, this error is maximal when the donor node is located in the middle of the receiver cell, and minimal when donor and receiver nodes are coincident. This alternated behavior is responsible for the generation of waves circled in Fig. 12(b). While the amplitude of the associated tone does not depend on the interpolation order at the interface, the high-order interpolation generates less noise in the spectrum and reduces waves amplitudes emanating from the interface (Fig. 12). In the case of the variation of element size at the interface, 


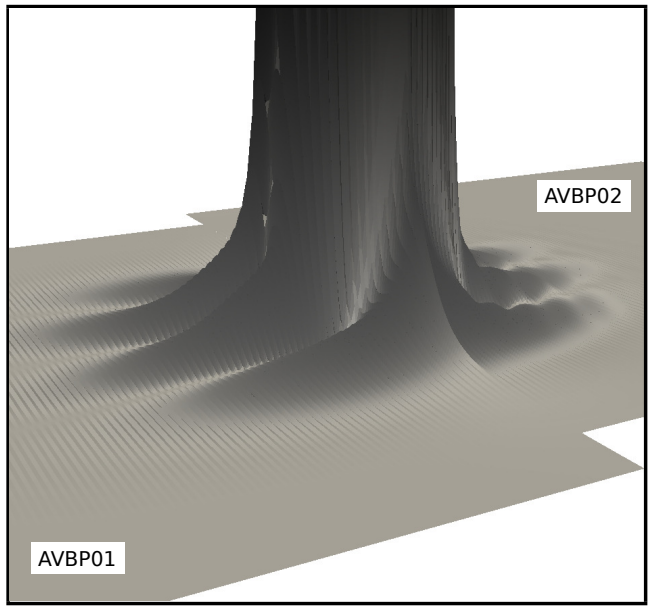

(a) I2 treatment at the interface.

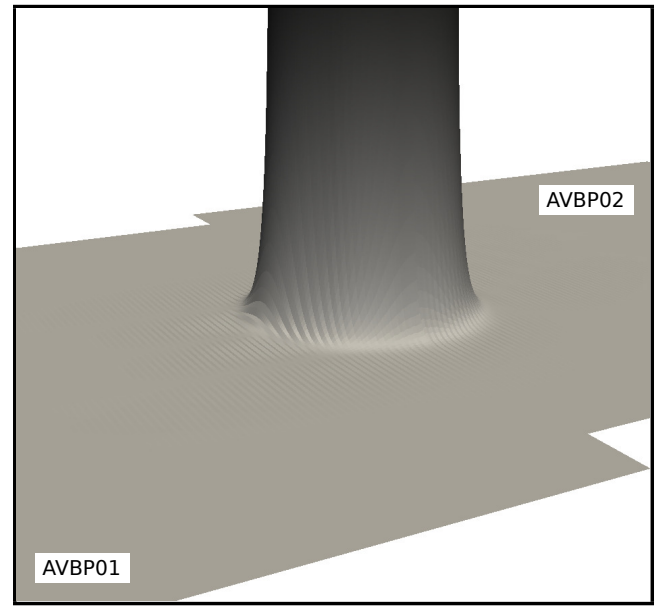

(b) I3 treatment at the interface.

Figure 13: Opposite of the pressure field at $t=0.5 T$ when the vortex crosses the interface. QSNMXY case, $U_{\text {grid }}=300 \mathrm{~m} / \mathrm{s}, N_{x, 01}=256$, TTGC scheme. Same color and amplitude scales for both figures.

two grid frequencies associated to the interpolation error on each domain can be defined:

$$
F_{\text {grid }, 01}=U_{\text {grid }} / \Delta y_{01} \quad \text { and } \quad F_{\text {grid }, 02}=U_{\text {grid }} / \Delta y_{02}
$$

Both frequencies are clearly visible in Fig. 14(b) for the QSDXY case with a moving interface, including higher harmonics. For all simulations with a moving interface in Figs. 14(a) and 14(b), a bump in the spectrum appears around $90 \mathrm{kHz}$, followed by other bumps. As already mentioned in [42], this phenomenon is caused by the temporal sampling of the grid period $T_{\text {grid }}=$ $1 / F_{\text {grid }}$. This sampling can be expressed, for the most detrimental case, as the ratio:

$$
\alpha_{t}=\frac{T_{\text {grid }}}{\Delta t}=\frac{\Delta y_{\text {min }}}{\Delta x_{\text {min }}} \frac{U_{0}+c_{0}+U_{\text {grid }}}{U_{\text {grid }} C F L} .
$$

Therefore a low value of $\alpha_{t}$ (here 3.7 for QSNMXY and QSDXY) implies a poor sampling of the grid period thus the presence of these bumps or aliasing frequencies in the spectra. In real applications (e.g. [45]), $\alpha_{t}$ is at least of the order of 20 so that no aliasing occurs. 


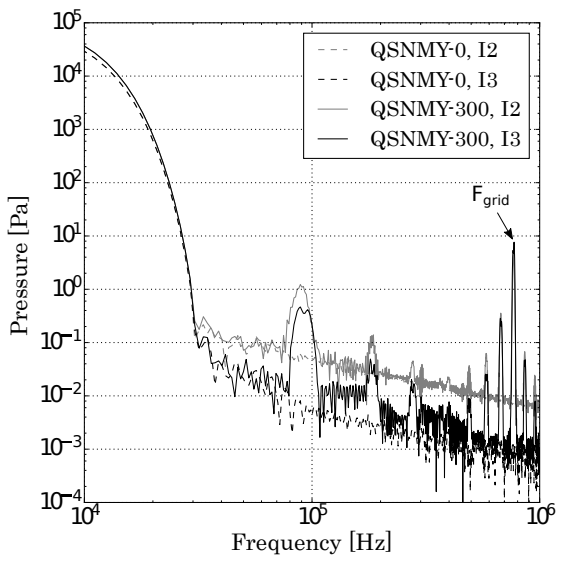

(a) QSNMXY case.

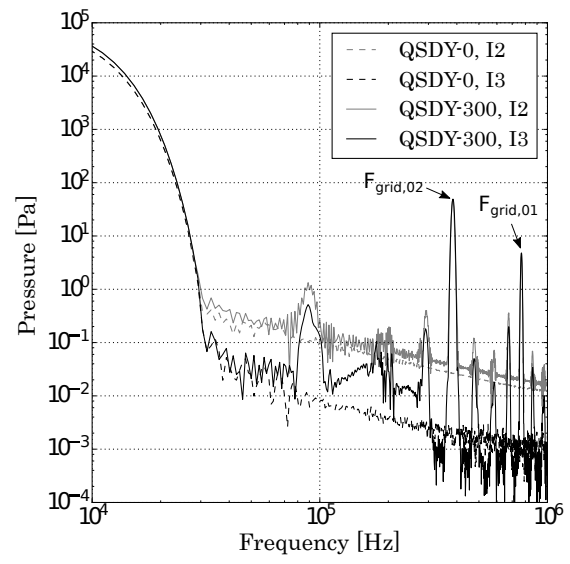

(b) QSDXY case.

Figure 14: FFT of the pressure signal recorded on the interface at $y_{c}, N_{x, 01}=256$, TTGC scheme.

To conclude, the simple test case of the 2-D inviscid vortex has allowed the evaluation of the interaction of MISCOG interpolation schemes with convective schemes. It has been shown that a spatial interpolation at the interface with the same order as the one of the convective scheme allows preserving the global order of accuracy of the simulation, even in the most detrimental cases: non-matching cells, coarsened cells in all directions and moving interface. This proves that the third order interpolation is efficient to preserve the high-order accuracy of the convective schemes of the solver. Three types of spurious waves have however been identified and are generated at the crossing of the interface by a vortical wave. The high-order overset method allows to effectively reduce amplitudes of these spurious waves, except for the tone associated with the interpolation error. In Navier-Stokes simulations, the laminar viscosity of the fluid is expected to damp spurious waves, justifying the need to consider a viscous vortex test case as discussed in the following section.

\section{Analysis of the overset grid method for a convected two-dimensional viscous vortex}

Following the evaluation of MISCOG with pure convective schemes, the behavior of the diffusive operators through the overlapping interface method 
is here assessed using the Navier-Stokes equations. In particular, the continuity of the derivatives of the velocity field should be ensured at the interface, as diffusive fluxes and SGS fluxes are based on these derivatives. The expected damping of the spurious waves by the flow viscosity needs also to be confirmed. These are the reasons why a $2-\mathrm{D}$ viscous vortex test case [66] is chosen here.

\subsection{Configurations}

The numerical set-up is identical to the one used in Sec. 4, except that the fluid viscosity is now non zero. Defining a Reynolds number based on the radius of the vortex $R_{c}$ as:

$$
R e=\frac{U_{0} R_{c}}{\nu},
$$

the kinematic viscosity is fixed such that $R e=1000\left(\nu=6.95 \times 10^{-4} \mathrm{~m}^{2} / \mathrm{s}\right)$. This configuration is again a direct numerical simulation. The analytical solution is not trivial anymore as the amplitude of the vortex decays during its convection due to the momentum diffusion.

Similarly to the inviscid configuration, the cases of Table 1 are considered in this viscous configuration. The LW, TTG4A and TTGC schemes have all been tested. No significant effect of the interface treatment has been found for the LW scheme in terms of global error, so that it is not presented in Sec. 5.2. As shown in Sec. 5.3, a finer analysis is required to detect an influence of the interpolation order of MISCOG on LW.

\subsection{Global evaluation}

Since no analytical solution exists for the present configuration, the reference data used to compute errors have been obtained using the matching interface case (QSM) on an even more refined grid $\left(N_{x, 01}=512\right)$ and the TTGC scheme. The mean quadratic error on the pressure is then computed similarly to Eq. (15). In Fig. 15, similar observations as for the inviscid case (Fig. 8) can be drawn, i.e. the spatial order of the numerical schemes TTG4A and TTGC is preserved with the high-order overset grid method, even with a moving interface. For a coarsening of cells at the crossing of the interface, Fig. 16 shows that this high-order interpolation allows increasing the order of simulations of a value comprised between 0.7 and 1 in comparison with the second-order interpolation, similarly to inviscid cases. As already encountered, the error due to the moving interface is larger with a cell coarsening than with a non-matching interface. 


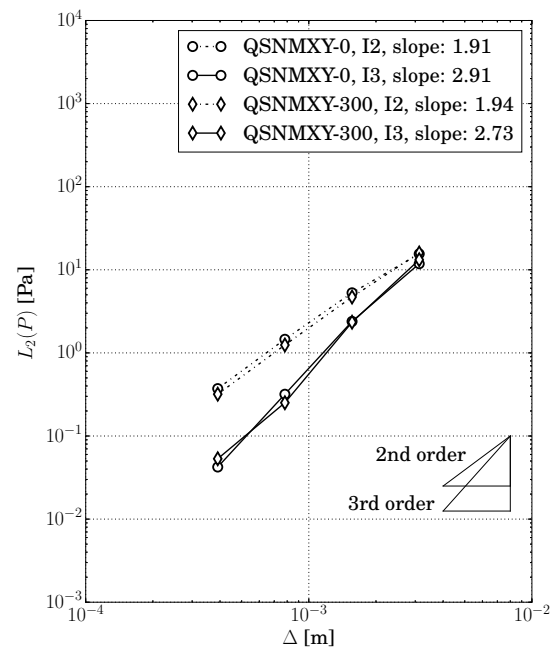

(a) TTG4A scheme.

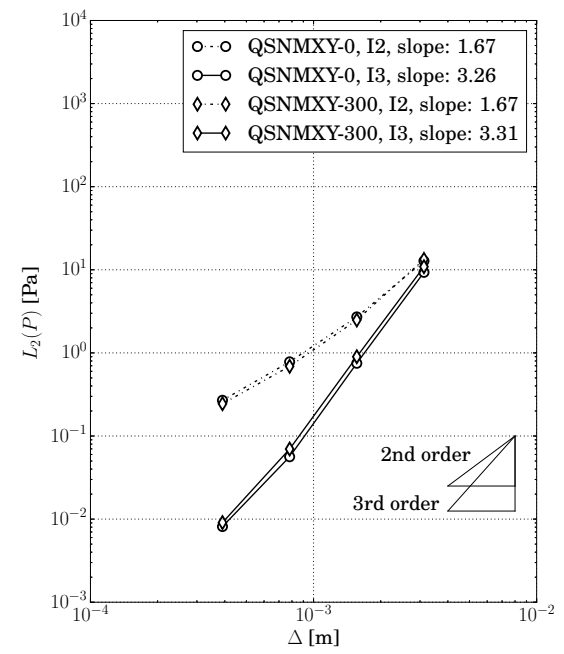

(b) TTGC scheme.

Figure 15: Quadratic error on the axial velocity, at time $t=T$, for a non-matching interface along $\mathrm{x}$ - and $\mathrm{y}$-direction (QSNMXY case), $\mathrm{Re}=1000$.

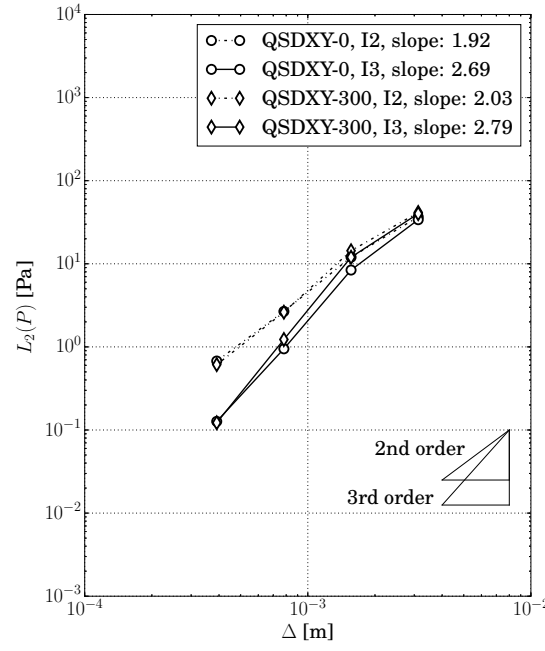

(a) TTG4A scheme.

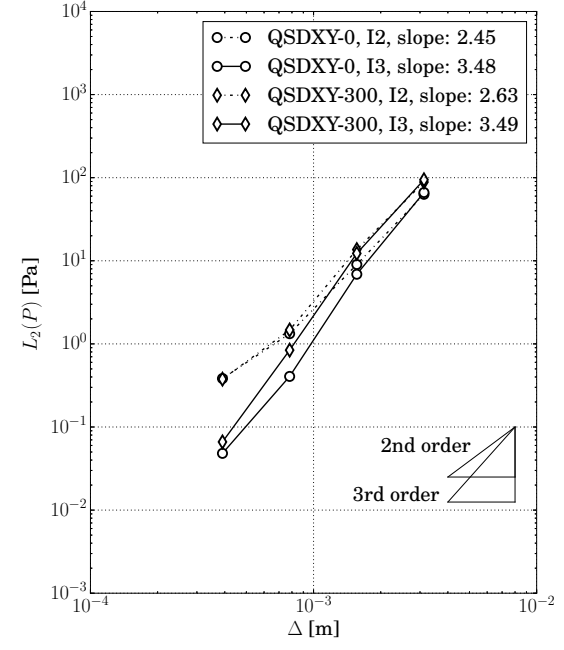

(b) TTGC scheme.

Figure 16: Quadratic error on the axial velocity, at time $t=T$, for AVBP02 coarsening along $\mathrm{x}$ and $\mathrm{y}$-directions (QSDXY case), $\mathrm{Re}=1000$. 


\subsection{Detailed evaluation}

To further evaluate MISCOG in this viscous context, Fig. 17 shows the difference of the simulated pressure with the REF pressure at $y=y_{c}$ for different instants. Just like before, this difference only illustrates the effect of the interface on the pressure signal. Only the curves corresponding to the TTGC scheme are presented as it is the most representative case. When comparing Figs. 17(a) and 17(b) with Figs. 12(a) and 12(b), the following observations can be made. First the physical acoustic waves generated when the vortex reaches the interface appear weakly influenced by the fluid viscosity, both in terms of amplitude and frequency. At this moderate value of the acoustic Knudsen number $\lambda^{2} f / \nu \approx 12000$, where $\lambda$ and $f$ are the wavelength and the frequency of the waves respectively, viscosity is indeed known to play a negligible role on acoustic waves over such a short distance [67]. As explained in Sec. 4.3, the numerical discontinuity is responsible for the Nyquist frequency waves seen in Figs. 17(a) and 17(b). However their amplitudes are lower at the time of their generation $t=0.5 \mathrm{~T}$ and their decay is clearly stronger than for the inviscid cases. Indeed no more Nyquist frequency waves are visible at $t=0.8 T$, whereas they are still present at the last instant of the simulation for the Euler flow (at least for the moving interface). The waves specifically generated by the moving interface (circled in Fig. 17) are also seen to be impacted by the fluid viscosity, since their amplitude is smaller than in the inviscid case. As anticipated, this section shows that fluid viscosity has a major effect on the Nyquist frequency waves generated at the interface and a significant influence on the waves due to the moving interface, since both are reduced by diffusion. For an actual LES, this behavior is beneficial since these spurious waves are reduced leading to a decrease of the error induced by the overset interface.

In addition, a study of the first derivatives of the velocity field is required as these are directly used within diffusive operators. In order to analyze the behavior of these derivatives through the interface, Fig. 18 focuses on the derivative of $V_{y}$ respectively to $y$ : $\partial V_{y} / \partial y$. The differences of the profiles, in each domain, with the corresponding REF profiles are plotted at $y=$ $0.52 L_{y}$ at the instant $t=0.5 T$ when the vortex crosses the interface. The LW scheme is presented here for the first time, since the interface treatment has a noticeable influence. Each domain is identified by different markers: crosses indicate the I2 treatment, circles and squares refer to the I3 treatment. For a fixed non-matching interface (Figs. 18(a), 18(b) and 18(c)), the improvement in the prediction of the velocity derivative is clearly highlighted 

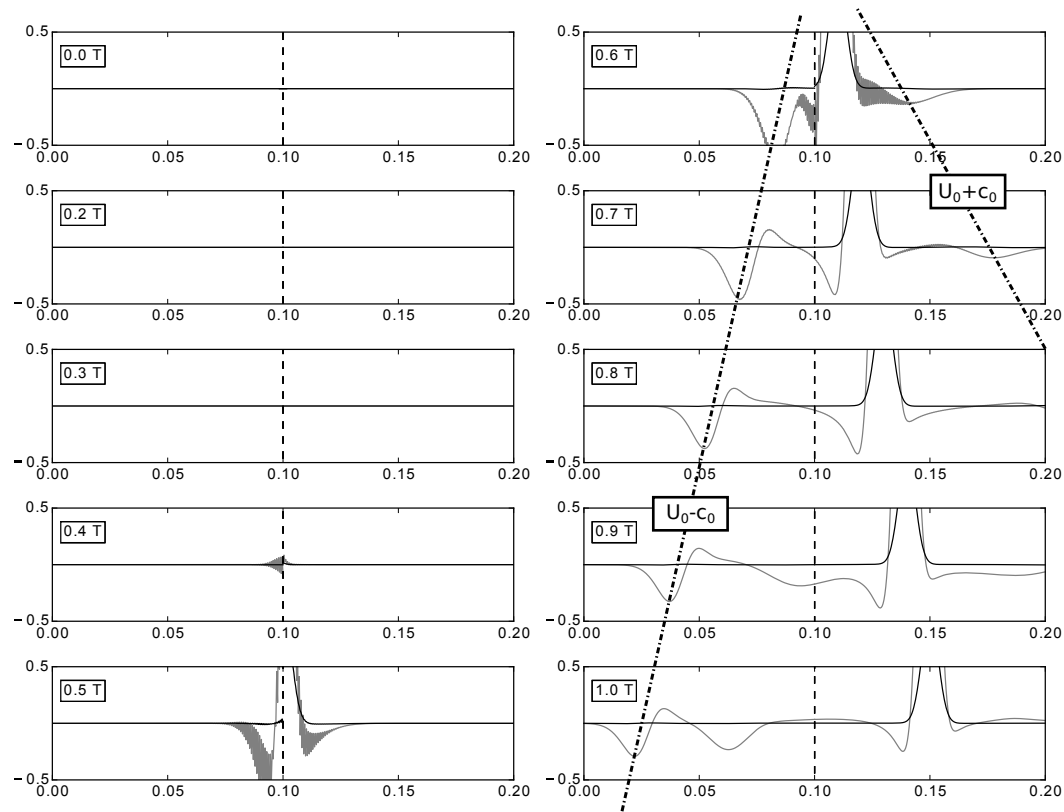

(a) TTGC scheme, $U_{\text {grid }}=0 \mathrm{~m} / \mathrm{s}$.
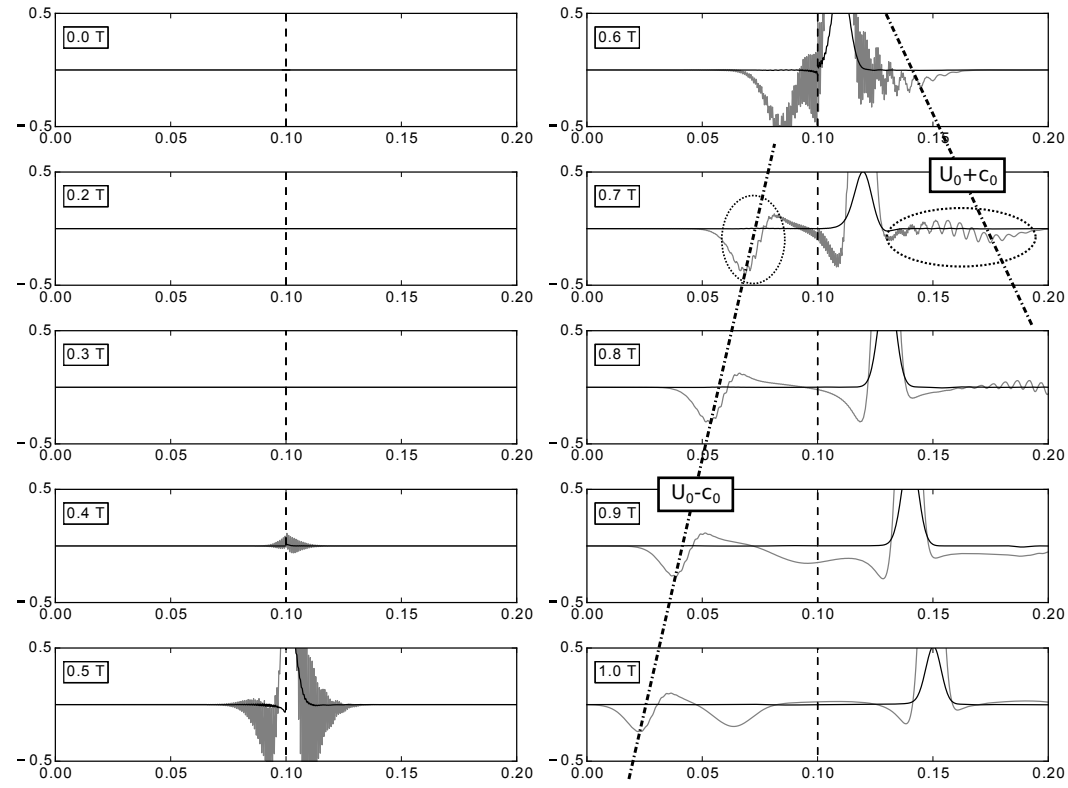

(b) TTGC scheme, $U_{\text {grid }}=300 \mathrm{~m} / \mathrm{s}$.

Figure 17: Pressure profiles, at $y=y_{c}$, at different instants between 0 and $T$, relative to the reference case $(\mathrm{REF}), N_{x, 01}=256, \mathrm{Re}=1000$. QSNMXY, TTGC scheme. Gray lines: second order interpolation at the interface. Black lines: third order interpolation at the interface. 
with the high-order interpolation. Indeed the latter drastically reduces the error away from the interface compared with the second order interpolation method. Moreover a high discontinuity in the derivatives appears with the I2 treatment right at the interface $\left(x / R_{c}=0\right)$. This is clearly detrimental for diffusive flux computations. This discontinuity is highly reduced with the high-order interpolation, from 94\% (LW) to 97\% (TTG schemes) relative to I2. For the cases with moving interfaces (Figs. 18(d), 18(e) and 18(f)), the errors relative to the REF solution are higher than with a fixed case, whatever the interpolation scheme at the interface. However the I3 overset method still allows decreasing this error. The most important observation concerns the reduction of the discontinuity of the derivative at the interface thanks to the high-order interpolation, from more that 50\% (LW) to $96 \%$ (TTG). This analysis demonstrates the interest of using the I3 treatment even with a second order scheme. This point will be further shown in Sec. 6 .
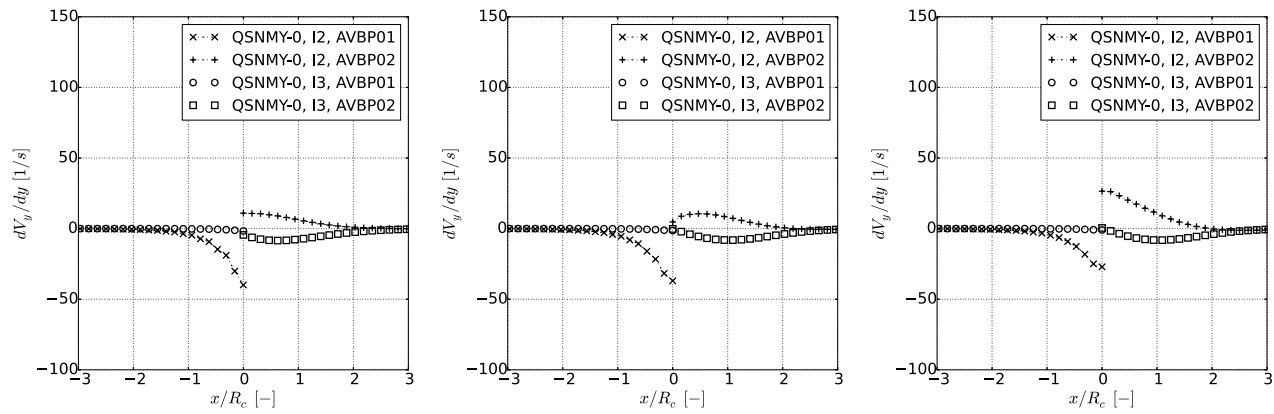

(a) LW, $U_{\text {grid }}=0 \mathrm{~m} / \mathrm{s}$.

(b) TTG4A, $U_{\text {grid }}=0 \mathrm{~m} / \mathrm{s}$.

(c) TTGC, $U_{\text {grid }}=0 \mathrm{~m} / \mathrm{s}$.
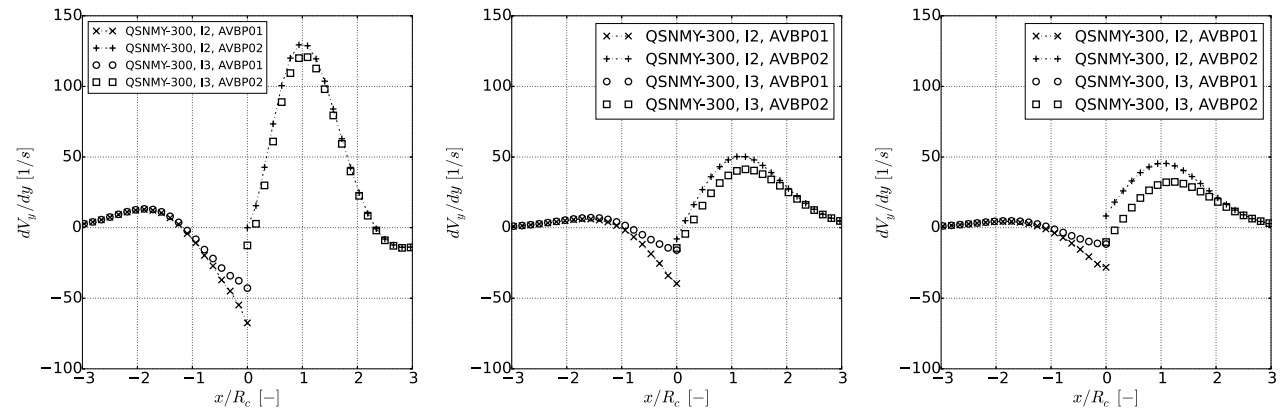

(d) $\mathrm{LW}, U_{\text {grid }}=300 \mathrm{~m} / \mathrm{s}$.

(e) TTG4A, $U_{\text {grid }}=300 \mathrm{~m} / \mathrm{s}$. (f) TTGC, $U_{\text {grid }}=300 \mathrm{~m} / \mathrm{s}$.

Figure 18: Differences of $\partial V_{y} / \partial y$ profiles with REF profiles, at $t=0.5 T$ and $y=0.52$ $L_{y}$, QSNMXY case, $N_{x, 01}=128, \operatorname{Re}=1000$. 
To conclude, similar results in terms of global spatial errors have been shown between inviscid and viscous cases. Namely the high-order overset method preserves the accuracy of the schemes for this configuration dominated by convection. Moreover the influence of the fluid viscosity on the spurious waves generated at the interface has been highlighted, as both Nyquist frequency waves and waves caused by a moving interface are efficiently damped. Finally it has been shown that the high-order interpolation is needed to properly transfer velocity derivatives across a fixed and a moving interface, even for a second order convective scheme. Using this high-order overset grid method is thus a prerequisite for the accurate simulation of diffusive fluxes and SGS fluxes in the targeted LES applications.

\section{Analysis of the overset grid method for a 3D viscous wake down- stream a cylinder}

Following the evaluation of MISCOG on the convective and diffusive operators in 2-D vortex cases, a 3-D LES needs to be considered. In particular, in actual turbomachinery applications, MISCOG interfaces are located between bladed rows and thus have to ensure the accurate propagation of blade wakes. To represent this configuration, the chosen test case consists of the viscous flow around a $3-\mathrm{D}$ circular cylinder. The influence of the interface, located in the wake, will be studied on this flow sensitive to transitions.

\subsection{Configurations}

The viscous flow around a 3-D circular cylinder has been widely studied both experimentally and numerically, over a large range of Reynolds numbers [68-71]. Defining the Reynolds number $R e_{D}$ based on the cylinder diameter $D$ and the free stream velocity $U_{0}$, a value of $R e_{D}=100$ is chosen. Indeed for this value of the Reynolds number, comprised between 40 and 190, the flow is laminar and it evidences a laminar vortex shedding (VS), called the Karman vortex street which develops downstream the cylinder [71]. This test case hence allows representing an unsteady and viscous wake that develops downstream a turbomachinery blade. The computational domain is shown in Fig. 19. It extends $15 D$ upstream to $45 D$ downstream the cylinder center. The simulation is three dimensional since the cylinder span equals 4 $D$. At the inlet, located on the left of the domain, a uniform axial velocity is imposed, with $U_{0}=50 \mathrm{~m} / \mathrm{s}$. The flow is initialized with uniform temperature and pressure of $300 \mathrm{~K}$ and $1.10^{5} \mathrm{~Pa}$ respectively. This pressure is also weakly 
imposed at the outlet (right boundary of Fig. 19). Both inlet and outlet conditions are treated with a characteristic formulation [64, 65]. Periodic boundary conditions are imposed on lateral boundaries, whereas the bottom and top boundaries are treated as slip adiabatic walls, so that no periodicity is enforced in the spanwise direction. As shown in Fig. 19, the MISCOG interface is located near the cylinder trailing edge, i.e. $2 D$ downstream the cylinder center. This corresponds to a quite detrimental case, that could be possibly encountered in a turbomachinery application, for which the interface should be located in the near wake of a blade. Tetrahedra cells are used to mesh the whole domain, except on the cylinder wall where 5 layers of prisms are present, giving a 12.5 millions cells grid. The measured $\mathrm{y}^{+}$value is less than one for the entire first layer of prisms, the other normalized dimensions being $\mathrm{x}^{+}=\mathrm{z}^{+}=4$. The WALE SGS model [50], introduced in Sec. 2.1, is used to represent the unresolved flow scales.

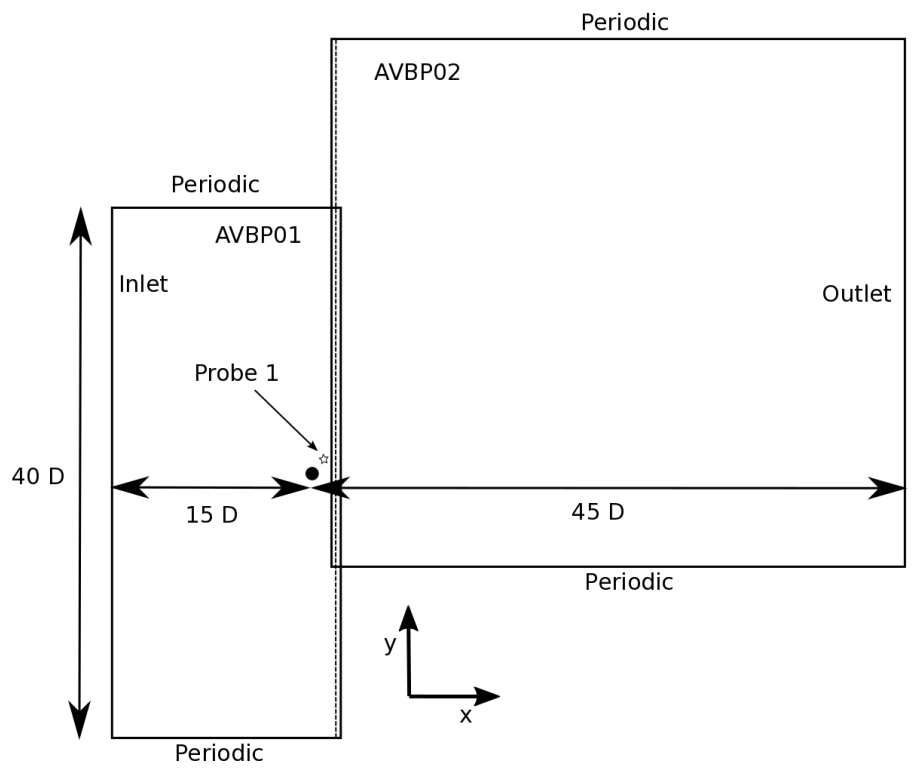

Figure 19: Sketch of the computational domain for the coupled case, in the $(x, y)$ plane. The domain extends $4 D$ in the z direction, where $D$ is the cylinder diameter. The interface, located $2 \mathrm{D}$ downstream the cylinder center, is schematically represented with the dashed line.

Similarly to the vortex test cases, several configurations are investigated. First, the reference configuration, denoted hereafter REF, consists of a single grid for the entire computational domain shown in Fig. 19. This single grid is 
then split into two pieces, and each domain is meshed independently so that the overlapping interface has non coincident cells. This case is called CPLNM-0. To evaluate the effects of a mobile interface on the wake, a translation at $U_{\text {grid }}=300 \mathrm{~m} / \mathrm{s}$ is imposed to the AVBP02 domain along the y-direction, giving the case CPL-300. Again, the second (I2) and the third order (I3) interpolations methods at the interface are evaluated for the different cases. Both the LW and TTG4A numerical schemes are alternatively employed in this configuration.

\subsection{Results and discussion}

In terms of physics, starting with a uniform initialization of the simulations, the flow is expected to destabilize and a periodic vortex shedding establishes. All the results are presented for this permanent flow regime. Figure 20 provides an instantaneous snapshot of the vorticity magnitude at $z=$ $2 D$, at the same time relative to the vortex shedding period, for the whole set of test cases. As expected, the Karman vortex street clearly appears. However no difference is actually visible between these cases, indicating the need for a finer evaluation of the influence of MISCOG on the cylinder wake.

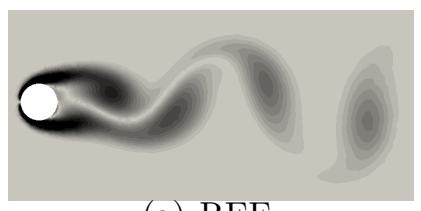

(a) REF.

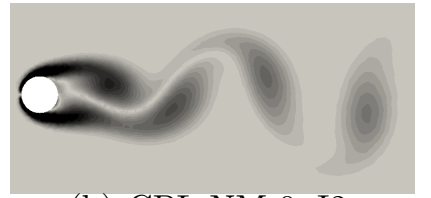

(b) CPL-NM-0, I2.

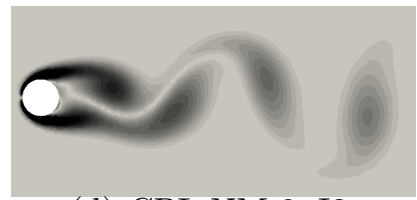

(d) CPL-NM-0, I3.

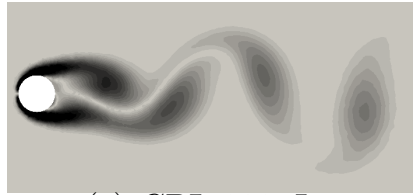

(c) CPL-300, I2.

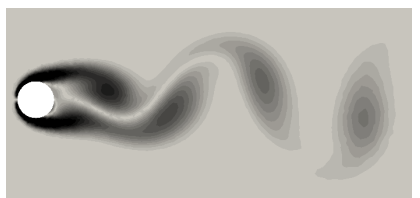

(e) CPL-300, I3.

Figure 20: Magnitude of the vorticity, at the same instant relative to the vortex shedding period. Cut at $z=2 D$, TTG4A scheme. Color scale varies from 0 to $30000 \mathrm{~s}^{-1}$.

Figure 21 shows the axial component of the velocity recorded at probe 1 , located at coordinates $(D, D, 2 D)$ (see Fig. 19), for flows simulated with TTG4A scheme. The time is made non-dimensional by the period of the vortex shedding. Starting from the same flow solution, a phase shift in the temporal signals appears for the cases with a second order interpolation 
(CPL-NM-0, I2 and CPL-300, I2) relative to the REF case. On the contrary, a third order interpolation at the interface seems to allow preserving the same frequency as the reference signal. This result shows that the second order interpolation at the interface generates perturbations that modify the flow upstream of the overlapping zone, potentially triggering a flow instability, both mechanisms being locked in time and resulting in a slightly modified unstable flow compared to the REF solution. This is in accordance with Secs. 4.3 and 5.3 that demonstrated the generation of spurious waves going back up the flow, with a larger amplitude for a I2 than for a I3 interface.

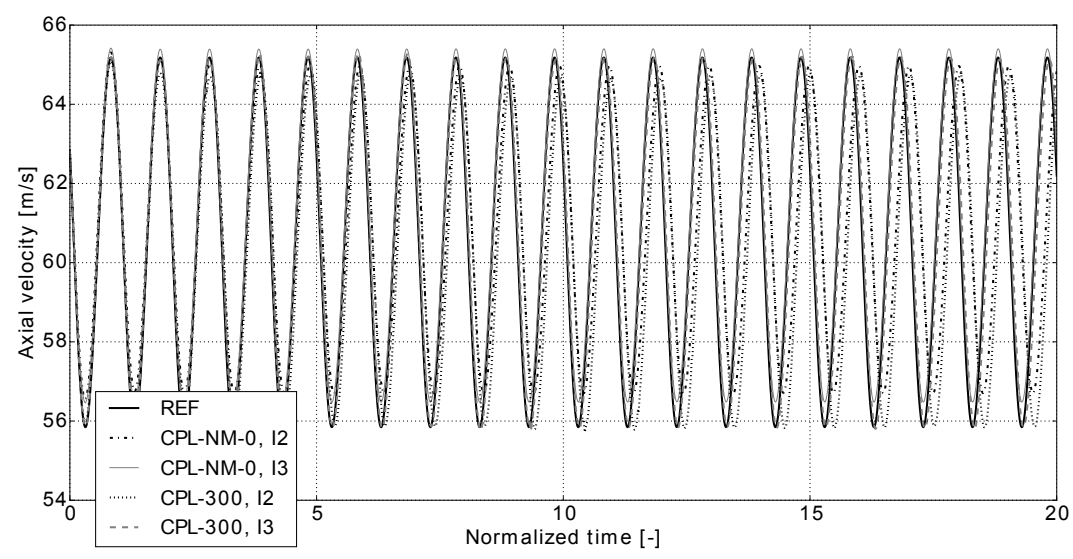

Figure 21: Temporal evolution of the axial velocity recorded at probe 1 (see Fig. 19), TTG4A scheme.

Axial velocity in probe 1 is then recorded during more than 40 periods, and the amplitude of its FFT is plotted in Fig. 22, for all the cases. Figure 22(a) corresponds to the cases simulated with LW scheme and Fig. 22(b) to the cases with TTG4A scheme. For both schemes, the REF case (plain black line) exhibits a peak at the vortex shedding frequency $F_{V S}$ as well as at its first harmonic. $F_{V S}$ is the same whatever the numerical scheme and is measured to be $F_{V S}=1638.7 \mathrm{~Hz}$. The corresponding Strouhal number, based on $D$ and $U_{0}$, is 0.164 . This value is in correct agreement with existing results from the literature at the same Reynolds number, e.g. Williamson [69] and Persillon and Braza [70], whose works predict a 0.164 Strouhal number. For each case of Figs. 22(a) and 22(b), the measured frequencies of the vortex shedding and its first harmonic are reported in Tables 2 and 3. Figure 22(a) and Table 2 show that for the LW scheme, when both domains are static, a third order interpolation at the interface allows to keep the fundamental and 


\begin{tabular}{lcc}
\hline \hline Case & Fundamental frequency $[\mathrm{Hz}]$ & First harmonic $[\mathrm{Hz}]$ \\
\hline \hline REF & $\mathbf{1 6 3 8 . 7}$ & $\mathbf{3 2 7 7 . 4}$ \\
CPL-NM-0, I2 & 1598.8 & 3237.4 \\
CPL-NM-0, I3 & $\mathbf{1 6 3 8 . 7}$ & $\mathbf{3 2 7 7 . 4}$ \\
CPL-300, I2 & 1598.8 & 3157.6 \\
CPL-300, I3 & 1598.8 & 3197.5 \\
\hline \hline
\end{tabular}

Table 2: Vortex shedding frequencies for the studied configurations, LW scheme.

\begin{tabular}{lcc}
\hline \hline Case & Fundamental frequency $[\mathrm{Hz}]$ & First harmonic $[\mathrm{Hz}]$ \\
\hline \hline REF & $\mathbf{1 6 3 8 . 7}$ & $\mathbf{3 2 7 7 . 4}$ \\
CPL-NM-0, I2 & $\mathbf{1 6 3 8 . 7}$ & 3237.4 \\
CPL-NM-0, I3 & $\mathbf{1 6 3 8 . 7}$ & $\mathbf{3 2 7 7 . 4}$ \\
CPL-300, I2 & 1598.8 & 3237.4 \\
CPL-300, I3 & $\mathbf{1 6 3 8 . 7}$ & $\mathbf{3 2 7 7 . 4}$ \\
\hline \hline
\end{tabular}

Table 3: Vortex shedding frequencies for the studied configurations, TTG4A scheme.

the first harmonic frequencies of the vortex shedding, whereas they are modified with the original second order interpolation. With a moving AVBP02 domain, an error appears in the vortex shedding frequency, but the errors on the VS amplitude and on the frequency of its first harmonic are decreased thanks to the third order interpolation. This case demonstrates the importance of using a third order interpolation at the interface even with a second order numerical scheme, as it allows to better predict amplitudes and frequencies of viscous wakes crossing the interface. For TTG4A (Fig. 22(b) and Table 3), even if the fundamental frequency is conserved with I2, its amplitude is far from the reference amplitude. On the contrary, the high-order interpolation at the interface gives the same fundamental and first harmonic frequency values for a static and a moving interface, as well as the correct amplitudes.

After having evaluated MISCOG on the convective and diffusive fluxes with the vortex cases, it is now of interest to study its influence on the subgrid scale content of the flow. To this end, the flow has been phaseaveraged according to the VS frequency at a random instant, and Fig. 23 presents the SGS stress tensor $\tau_{x y}^{t}$ (see Eq. (2)), out of which the value of 


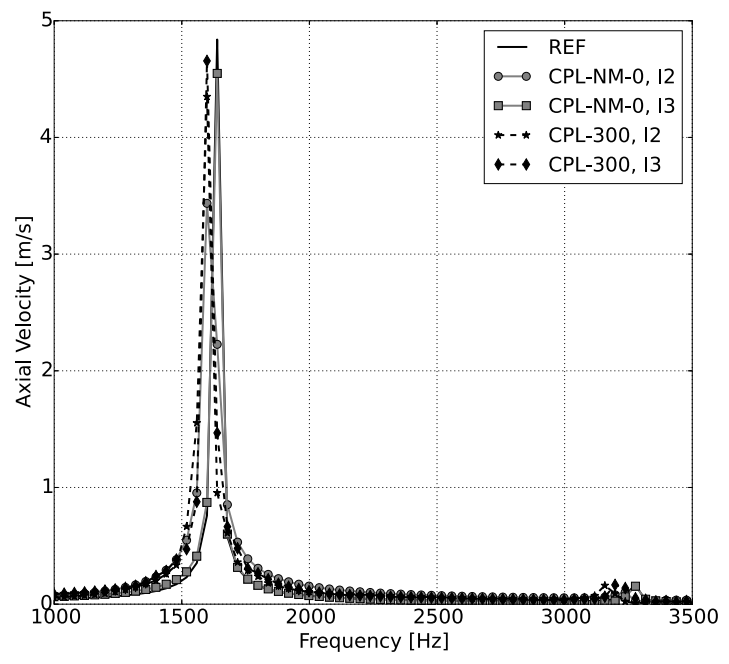

(a) LW scheme.

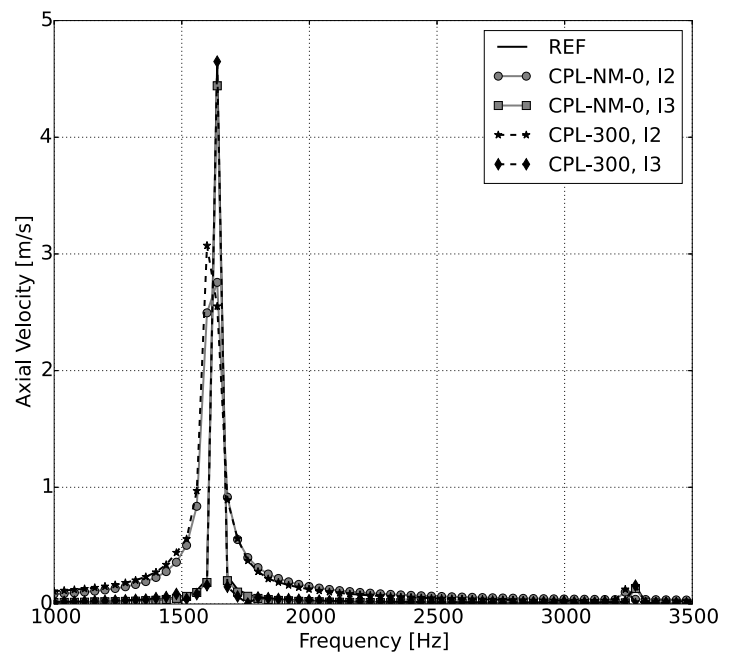

(b) TTG4A scheme.

Figure 22: Absolute values of the FFT of the axial velocity signal recorded at probe 1 (see Fig. 19). 
$\tau_{x y}^{t}$ obtained in the REF simulation has been removed. The comparison of Figs. 23(a) and 23(b) therefore actually measures the effect of MISCOG on the SGS tensor. A discontinuity of $\tau_{x y}^{t}$ appears at the interface, and is more pronounced in the case with the second order interpolation than in the case with the third order interpolation. Downstream the interface, it is worth noting the higher error of $\tau_{x y}^{t}$ in the wake with I2 compared to the I3 interface. This is consistent with results of Sec. 5.3 showing a more accurate transfer of velocity derivatives with I3, thus a better prediction of the SGS tensor.
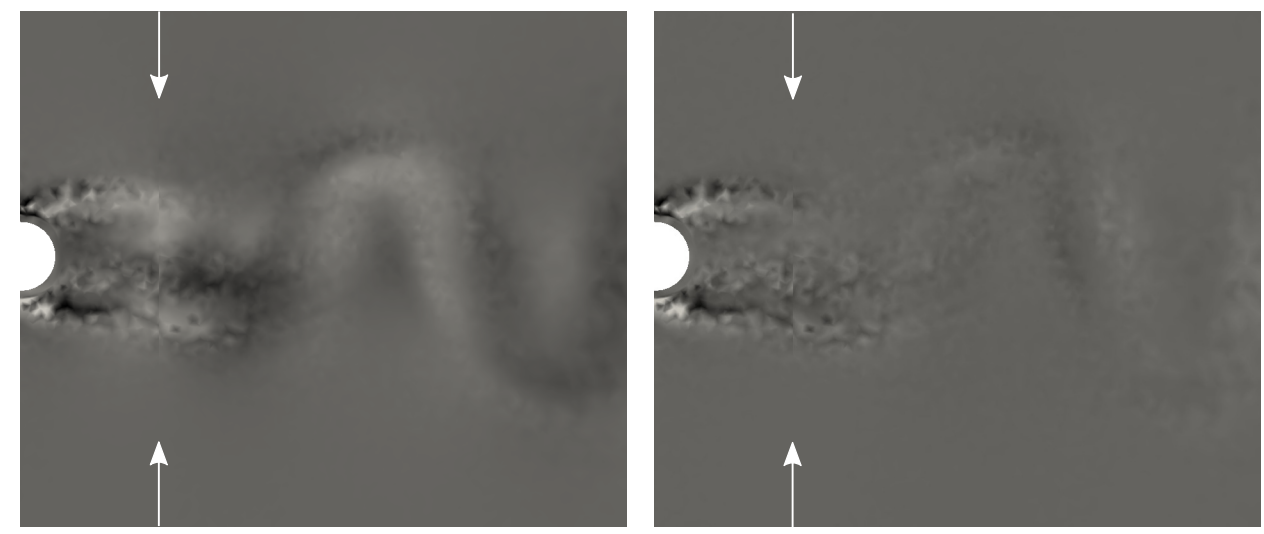

(a) Interface with second order interpola- (b) Interface with third order interpolation.

tion.

Figure 23: Case CPL-NM-0, TTG4A scheme. Difference between phase-averaged $\tau_{x y}^{t}$ and phase-averaged $\tau_{x y}^{t}$ from the REF case. White arrows indicate the location of the interface. Color scale varies from -10 to $10 \mathrm{~Pa}$.

To quantitatively evaluate the effect of MISCOG on the wake, Fig. 24 represents the phase-averaged wake profiles, at a random instant, in terms of axial velocity and SGS tensor, extracted right on the interface. In Fig. 24(a), only the second order interpolation with a moving interface provides a non negligible error on the axial velocity relative to the reference solution (black line). For the SGS tensor (Fig. 24(b)), both cases with an I2 interface yields an error. In addition, Fig. 24 illustrates that the high-order overset method allows preserving velocity and SGS tensors predicted by the reference TTG4A simulation.

\subsection{Mass conservation through overset grids}




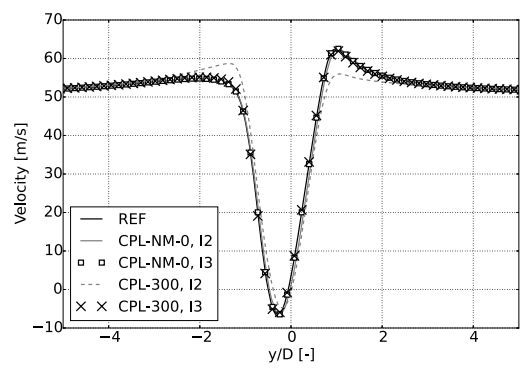

(a) Axial velocity.

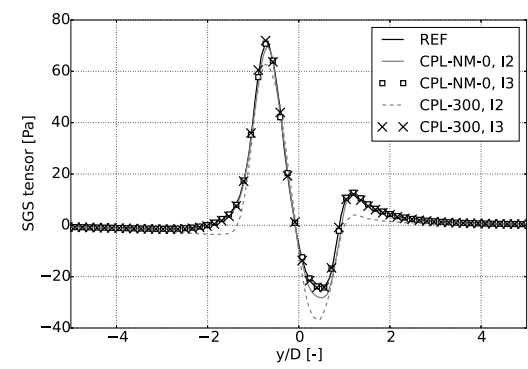

(b) SGS tensor $\tau_{x, y}^{t}$.

Figure 24: Phase-averaged profiles, at a random instant, in the transverse direction, on the interface $(x=2 \mathrm{D}, z=2 \mathrm{D})$, for TTG4A scheme.

This 3-D LES test case is a good candidate to evaluate mass conservation through the overlapping interface. Indeed, as explained in Sec. 2.2, MISCOG is not a conservative method, similarly to any overset grid method without conservative interpolation. The instantaneous mass flows $Q_{01}(t)$ and $Q_{02}(t)$ are computed in each domain AVBP01 and AVBP02 respectively, right on the interface plane $(x=2 D)$. The mass flow difference between both instances is then defined as:

$$
\Delta Q(t)=\frac{\left|Q_{01}(t)-Q_{02}(t)\right|}{\bar{Q}_{01}}
$$

where $\bar{Q}_{01}$ is the time-averaged mass flow computed in AVBP01. Figure 25 presents the temporal evolution of $\Delta Q(t)$ for static and moving interfaces, according to the interpolation order of MISCOG. It is shown that for all cases plotted, the relative difference in mass flow is negligible since less than $0.002 \%$. Nevertheless increasing the interpolation order at the interface has a positive influence on conservative properties since this decreases mass flow differences between both domains, of an approximate mean value of $30 \%$ for both cases.

\section{Analysis of the overset grid method for a strong shock}

Although the MISCOG stability is partly addressed through the previous set of test cases, a more stringent configuration seems needed for an adequate evaluation of the high-order interpolation scheme in this specific context: namely in the presence of discontinuities within the flow field. To this end, 


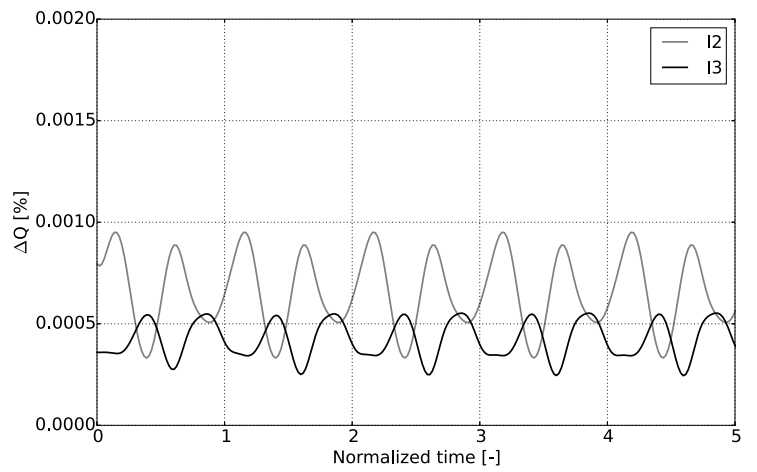

(a) CPL-NM-0.

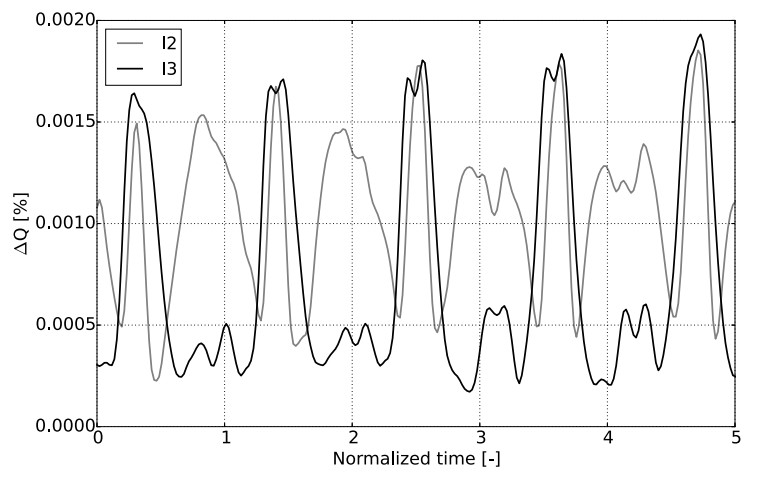

(b) CPL-300.

Figure 25: Temporal evolution of mass flow differences between AVBP01 and AVBP02 domains, computed on the interface at $x=2 D$, according to Eq. (21). TTG4A scheme. 
the test case selected corresponds to a blunt body placed into a hypersonic flow. For this specific problem, a strong steady bow shock is expected to establish upstream of the body. This case has already been used to evaluate overset grid method, e.g. in [36]. The flow is here simplified to a monospecies fluid. The computational domain is presented in Fig. 26. It consists of a quarter of cylinder of diameter $D=1 \mathrm{~m}$. The inlet is located $0.5 D$ upstream of the cylinder, and the outlet at the axial location of the cylinder center. This domain is extruded along z-direction of $0.01 D$. For the purpose of the study, this domain is split in two parts, around the interface located at $0.25 D$ upstream of the cylinder. Both AVBP01 and AVBP02 domains are meshed independently with tetrahedra cells, except close to the cylinder wall where 10 layers of prisms are imposed. As shown in Fig. 26, tetrahedra cells are not coincident in the overlapping region, their sizes being similar between both domains. Note that this mesh is not refined enough to precisely capture the shock, the objective being only to evaluate the capacity of the overset method to handle the strong shock.

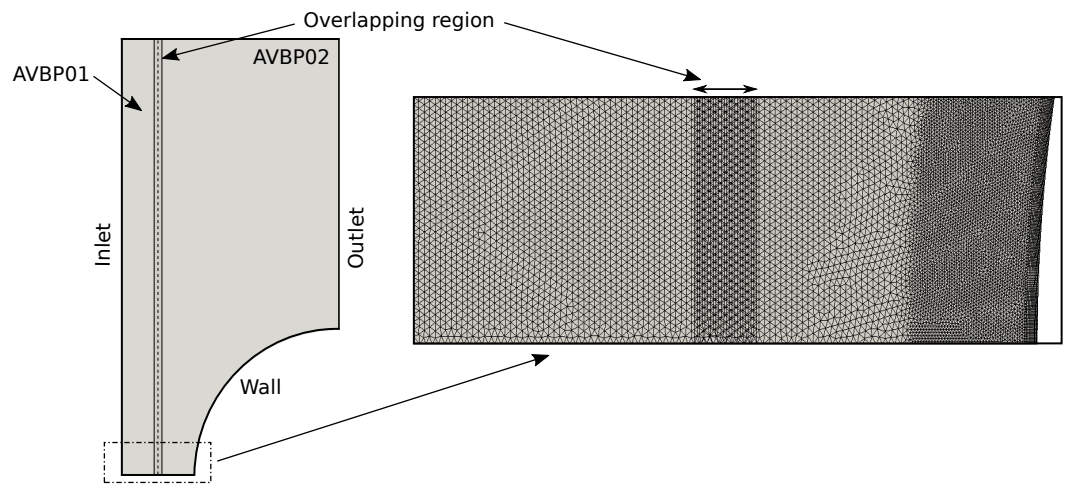

Figure 26: Computational domains for the shock test case, and zoom on the grid around the overset region.

At the supersonic inlet condition, the flow is imposed in the axial direction with $U_{0}=5000 \mathrm{~m} / \mathrm{s}, P_{0}=57.36 \mathrm{~Pa}$ and $T_{0}=200 \mathrm{~K}$. This yields a 19.3 Mach number flow. The simulations are performed with the LW scheme at $\mathrm{CFL}=0.7$, using a shock capturing technique [72]. Starting from a uniform solution, the shock establishes at a distance from the body crossing the MISCOG interface. A convergence study has then been performed to confirm the steady position of the shock. Figure 27 represents iso-lines of pressure computed with TurboAVBP, using I2 or I3 interpolation at the interface. 
The shock, identified by a strong pressure gradient, crosses the overlapping region at around $y=0.72 D$ (see arrows). Pressure contours appear smooth at the crossing of the overset grids, and no difference is visible between I2 and I3 interpolation methods at the interface.

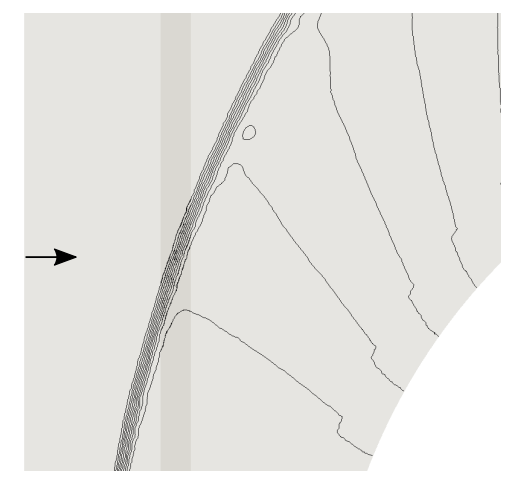

(a) I2 treatment at the interface.

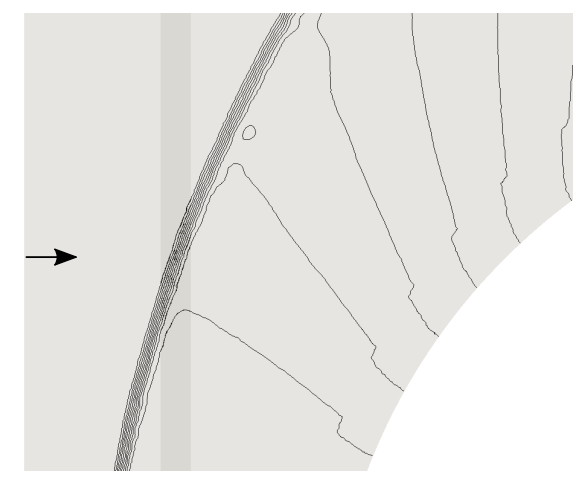

(b) I3 treatment at the interface.

Figure 27: Iso-lines of pressure across the overset region, on a planar cut at $z=0 D$. Lines are separated by $2000 \mathrm{~Pa}$, going from 2000 to $22000 \mathrm{~Pa}$. The arrow indicates the position $y=0.72 D$. The dark strip corresponds to the overlapping region.

To evaluate more precisely the influence of the interface treatment on a strong discontinuity, Figs. 28 and 29 show pressure and temperature profiles respectively, extracted at $y=0.72 D$ and $z=0 D$. The second-order interface is represented with dashed lines, and the high-order method with plain lines. Grey and black colors indicate AVBP01 and AVBP02 domains respectively. Globally, in Figs. 28(a) and 29(a), the dashed and plain lines are superimposed, showing that the order of the interface treatment has no influence on the shock predictions, as well as the upstream and downstream fields. Slight differences can be noticed within the shock in Figs. 28(b) and 29(b), but are observed to be negligible.

Note that such a strong shock, with a pressure difference of $19 \mathrm{kPa}$, is not expected to occur in turbomachinery applications targeted to be used with TurboAVBP. Nevertheless this section demonstrates the capability of MISCOG, with basic and high-order interpolation methods, to successfully transfer a strong shock, when a shock capturing technique is employed. As a consequence, the high-order overset grid method is expected to be stable for turbomachinery applications, where discontinuities of smaller amplitudes can be found. 


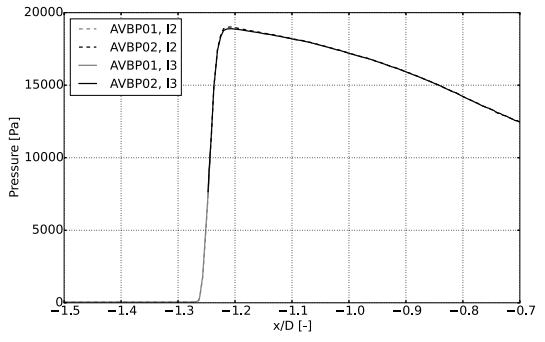

(a) Entire domain upstream of wall.

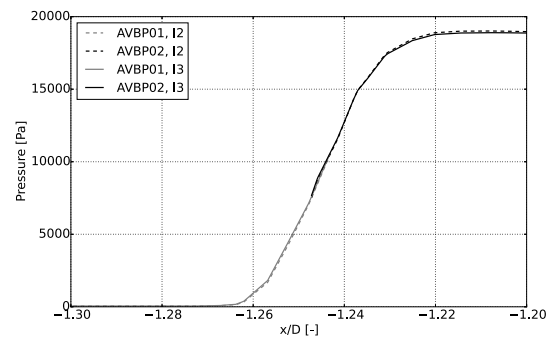

(b) Zoom around overset grid region.

Figure 28: Pressure profile at $y=0.72 D$ and $z=0 D$, for both interface treatments.

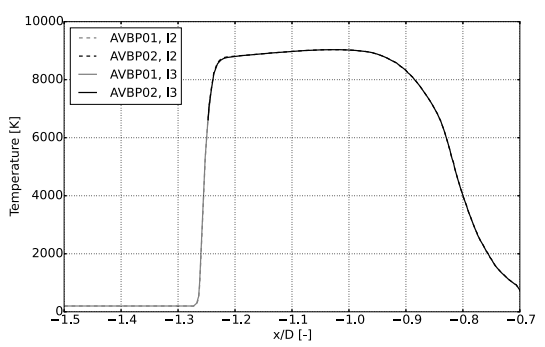

(a) Entire domain upstream of wall.

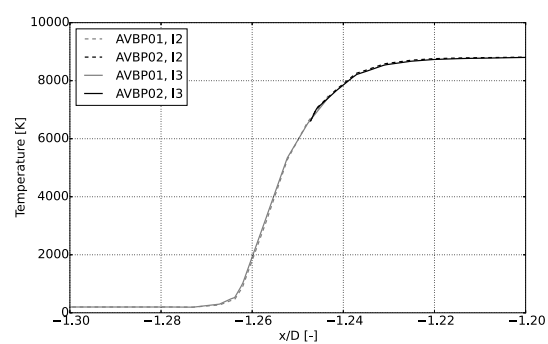

(b) Zoom around overset grid region.

Figure 29: Temperature profile at $y=0.72 D$ and $z=0 D$, for both interface treatments. 


\section{Conclusion}

This paper proposed an analysis of the overset grid method MISCOG aiming at being used along with the unstructured LES solver TurboAVBP designed to be applied to turbomachinery stages. The latter requires an accurate transfer of flow variables across the interfaces to limit dissipative and dispersive errors on the vortical, acoustic and entropic waves typically found in such simulations. That is the reason why a high-order interpolation method has been introduced and systematically studied throughout the entire paper. This interpolation relies on nodal gradients available in the solver and 1-D Hermite interpolators. First, it has been demonstrated that the implemented high-order interpolation is at least third order accurate in space for several types of 2-D and 3-D elements. Then the study has focused on the interactions of MISCOG with flow problems of increasing complexity. The test case of an inviscid vortex convected through the interface has allowed the evaluation of the influence of MISCOG on third order convective schemes. It has been shown that the high-order interpolation preserves the global accuracy of the schemes, even in the most detrimental case: non-matching cells at the interface, coarsened cells in all directions and moving interface. In addition, three types of spurious waves generated at the interface, representing a numerical discontinuity for the flow, have been identified. They consist of physical waves, i.e. acoustic waves, numerical waves, i.e. Nyquist frequency waves, and waves only present with a moving interface since they are caused by the temporally evolving spatial interpolation error. The high-order interpolation has been shown to significantly reduce these waves respectively to the original second order accurate interpolation method in MISCOG.

Following this Euler configuration, the case of a convected viscous vortex has been chosen for two main reasons. As expected, it has been observed that the laminar flow viscosity damps the spurious waves generated at the interface. More precisely, only the Nyquist frequency waves and the waves due to a moving interface are affected by the flow viscosity, which is beneficial for the global accuracy of MISCOG. Then a study of velocity derivatives through the interface has shown that the high-order interpolation is required to properly transfer diffusive fluxes, for second and third order convective schemes. Indeed a second order interpolation method systematically yields discontinuities of velocity derivatives at the interface.

The case of the 3-D viscous wake downstream a circular cylinder has then been presented. By inserting a MISCOG interface in the near wake, 
the flow problem is similar to an actual turbomachinery application where the interface is located between two bladed rows. A simulation without any interface has first been validated in terms of Strouhal number respectively to results from the literature. It has been shown that only the high-order overset method keeps the frequency content of the wake, whereas the second order method disturbs the flow, even in the upstream direction, due to spurious waves generated at the interface. The interest of using the high-order interpolation even with a second-order scheme has been demonstrated, as this is required to accurately transfer the wake through the interface. The SGS tensor has also been shown to be accurately handled with the high-order overset method. The mass conservation property of MISCOG has been evaluated on this case. The high-order interpolation allows decreasing the mass flow error between both instances by around 30\%. When measuring the computational cost on this 3-D test case, it has been confirmed that this high-order method is computationally efficient since it has the same cost as the second order method, for static and moving interfaces.

Finally, the extreme case of an hypersonic flow has addressed the stability behavior of the proposed high-order overset grid method. It has been shown that this method is able to handle a shock of $19 \mathrm{kPa}$ pressure difference, without any visible discrepancy in comparing with the second order overset method. Even if such a strong discontinuity is not expected to occur in turbomachinery applications for TurboAVBP, MISCOG appears as a robust method for complex flow problems.

To conclude, a high-order interpolation scheme has been correctly implemented within MISCOG and is shown to be third order accurate. From the test cases studied in this paper, it is highly recommended to use this method whatever the numerical convective scheme, for the following demonstrated reasons: it keeps the global accuracy of the scheme; it limits amplitudes of spurious waves generated at the interface; it accurately transfers diffusive fluxes and SGS tensor and its cost is identical to the one of the original method. Actual turbomachinery cases are currently simulated and confirm these conclusions. Current perspectives and potential subject of interest still to be probed today are the effective impact of the SGS discontinuity on a LES prediction and the risk behind the spurious numerical waves generated at the moving interface. 


\section{Acknowledgements}

The authors wish to acknowledge the foundation of Sciences et Technologies pour l'Aeronautique et l'Espace for its financial support within the RTRA project COFFECI, as well as GENCI for the computational resources provided within the allocations x20152a6074 and x20162a6074. The authors are also grateful to MITACS organization for its financial support through the Elevate fellowship.

\section{References}

[1] S. B. Pope, Turbulent flows, Cambridge University Press, 2000.

[2] P. Sagaut, Large Eddy Simulation for incompressible flows, Springer, 2002.

[3] M. Lesieur, O. Métais, P. Comte, Large-Eddy Simulations of Turbulence, Cambridge University Press, 2005.

[4] T. Poinsot, D. Veynante, Theoretical and Numerical Combustion, Third Edition (www.cerfacs.fr/elearning), 2011.

[5] P. Tucker, Computation of unsteady turbomachinery flows: Part 2 LES and hybrids, Prog. Aerospace Sci. 47 (7) (2011) 546 - 569.

[6] W. McMullan, G. Page, Towards large eddy simulation of gas turbine compressors, Prog. in Astronautics and Aeronautics 52 (0) (2012) 30 47.

[7] N. Gourdain, F. Sicot, F. Duchaine, L. Gicquel, Large eddy simulation of flows in industrial compressors: a path from 2015 to 2035, Proc. R. Soc. Lond. A, Mathematical and Physical Sciences.

[8] J. C. Tyacke, P. G. Tucker, Future use of large eddy simulation in aeroengines, J. Turbomach. 137 (2015) 1-16.

[9] G. Fillola, M.-C. Lepape, M. Montagnac, Numerical simulations around wing control surfaces, in: Proceedings of 24th International Congress of the Aeronautical Sciences, Yokohama, Japan, 2004. 
[10] B. Francois, M. Costes, G. Dufour, Comparison of chimera and sliding mesh techniques for unsteady simulations of counter rotating openrotors, in: 20th ISABE, 2011.

[11] V. Ganine, D. Amirante, N. Hills, Enhancing performance and scalability of data transfer across sliding grid interfaces for time-accurate unsteady simulations of multistage turbomachinery flows, Comput. Fluids 115 (2015) 140-153.

[12] H. A. Schwarz, Ueber einige abbildungsaufgaben, Journal fur die Reine und Angewandte Mathematik 70 (1869) 105-120.

[13] E. A. Volkov, The method of composite meshes for finite and infinite regions with piecewise smooth boundary, Proc. Steklov Inst. Math. 96 (1968) 145-185.

[14] G. Starius, Composite mesh difference methods for elliptic and boundary value problems, Numer. Math. 28 (1977) 243-258.

[15] E. H. Atta, J. Vadyak, A grid overlapping scheme for flowfield computations about multicomponent configurations, AIAA Journal 21 (1983) 1271-1277.

[16] J. Benek, J. Steger, F. Dougherty, A flexible grid embedding technique with application to the Euler equations, AIAA Paper 83-1944.

[17] M. J. Berger, On conservation at grid interfaces, SIAM Journal on Applied Mathematics 24 (1987) 967-984.

[18] W. D. Henshaw, G. S. Chesshire, Multigrid on composite meshes, SIAM J. Sci. Stat. Comput. 8 (6) (1987) 914-923.

[19] G. S. Chesshire, W. D. Henshaw, Composite overlapping meshes for the solution of partial differential equations, J. Comput. Phys. 90 (1) (1990) $1-64$.

[20] Z. J. Wang, A fully conservative interface algorithm for overlapped grids, J. Comput. Phys. 122 (1) (1995) 96-106.

[21] W. Henshaw, D. Schwendeman, An adaptive numerical scheme for highspeed reactive flow on overlapping grids, J. Comput. Phys. 191 (2) (2003) 420-447. 
[22] M. C. Galbraith, J. A. Benek, P. D. Orkwis, M. G. Turner, A discontinuous Galerkin chimera scheme, Comput. Fluids 98 (2014) 27-53.

[23] B. E. Merrill, Y. T. Peet, P. F. Fischer, J. W. Lottes, A spectrally accurate method for overlapping grid solution of incompressible NavierStokes equations, J. Comput. Phys. 307 (2016) 60-93.

[24] M. J. Brazell, J. Sitaraman, D. J. Mavriplis, An overset mesh approach for 3D mixed element high-order discretizations, J. Comput. Phys. 322 (2016) 33-51.

[25] G. Desquesnes, M. Terracol, E. Manoha, P. Sagaut, On the use of a high order overlapping grid method for coupling in CFD/CAA, J. Comput. Phys. 220 (1) (2006) 355-382.

[26] D. J. Bodony, G. Zagaris, A. Reichert, Q. Zhang, Provably stable overset grid methods for computational aeroacoustics, J. Sound Vib. 330 (11) (2011) 4161-4179.

[27] W. Henshaw, K. A. Chand, A composite grid solver for conjugate heat transfer in fluid-structure systems, J. Comput. Phys. 228 (10) (2009) $3708-3741$.

[28] D. S. William, W. D. Henshaw, Moving overlapping grids with adaptive mesh refinement for high-speed reactive and non-reactive flow, J. Comput. Phys. 216 (2) (2006) 744-779.

[29] S. Volkner, X. Luo, W. R. Wriggers, T. Rung, An overset-grid threephase flow model for offshore operations, in: VI International Conference on Computational Methods in Marine Engineering, 2015.

[30] L. Castillon, G. Legras, Overset grid method for simulation of compressors with nonaxisymmetric casing treatment, J. Prop. Power 29 (2) (2013) 460-465.

[31] L. Castillon, G. Billonnet, J. Riou, S. Peron, C. Benoit, A technological effect modeling on complex turbomachinery applications with an overset grid numerical method, J. Turb. 136.

[32] C. K. W. Tam, K. A. Kurbatskii, A wavenumber based extrapolation and interpolation method for use in conjunction with high-order finite difference schemes, J. Comput. Phys. 157 (2000) 588-617. 
[33] H. S. Tang, S. C. Jones, F. Sotiropoulos, An overset-grid method for 3D unsteady incompressible flows, J. Comput. Phys. 191 (2003) 567-600.

[34] S. E. Sherer, J. N. Scott, High-order compact finite-difference methods on general overset grids, J. Comput. Phys. 210 (2) (2005) 459 - 496.

[35] T. K. Sengupta, V. Suman, N. Singh, Solving Navier-Stokes equation for flow past cylinders using single-block structured and overset grids, J. Comput. Phys. 229 (1) (2010) 178-199.

[36] A. Lani, B. Sjogreen, H. C. Yee, W. D. Henshaw, Variable high-order overset grid methods for mixed steady and unsteady multiscale viscous hypersonic nonequilibrium flows, in: Annual Research Briefs, Center for turbulence Research, Stanford, 2011.

[37] J. Crabill, A. Jameson, J. Sitaraman, A high-order overset method on moving and deforming grids, in: AIAA Modeling and Simulation Technologies Conference, 2016.

[38] X. X. Chen, X. Zhang, High-order interface for aeroacoustic computation using overset grid, AIAA Journal 51 (2013) 519-523.

[39] B. Roget, J. Sitaraman, Robust and efficient overset grid assembly for partitioned unstructured meshes, J. Comput. Phys. 260 (2014) 1-24.

[40] S. Hahn, G. Iaccarino, S. Ananthan, J. D. Baeder, Extension of CHIMPS for unstructured overset simulation and high-order interpolation, in: Annual Research Briefs, Center for turbulence Research, Stanford, 2009.

[41] R. Shenoy, M. J. Smith, M. A. Park, Unstructured overset mesh adaptation with turbulence modeling for unsteady aerodynamic interactions, J. Aircraft 51 (2014) 161-174.

[42] G. Wang, F. Duchaine, D. Papadogiannis, I. Duran, S. Moreau, L. Y. M. Gicquel, An overset grid method for large eddy simulation of turbomachinery stages, J. Comput. Phys. 274 (2014) 333-355.

[43] T. Leonard, M. Sanjose, S. Moreau, F. Duchaine, Large eddy simulation of a scale-model turbofan for fan noise source diagnostic, in: 22nd AIAA/CEAS Aeroacoustics Conference, 2016. 
[44] G. Wang, S. Moreau, F. Duchaine, J. de Laborderie, L. Gicquel, LES investigation of aerodynamics performance in an axial compressor stage, in: 22nd Conference of the CFD Society of Canada, Toronto, Canada, 2014 .

[45] J. de Laborderie, F. Duchaine, O. Vermorel, L. Gicquel, S. Moreau, Application of an overset grid method to the large eddy simulation of a high-speed multistage axial compressor, in: ASME Turbo Expo, no. GT2016-56344, Seoul, South Korea, 2016.

[46] D. Papadogiannis, G. Wang, S. Moreau, F. Duchaine, L. Gicquel, F. Nicoud, Assessment of the indirect combustion noise generated in a transonic high-pressure turbine stage, J. Eng. Gas Turb. and Power 138 (4) (2015) 0415030 (8 pp.).

[47] R. Vichnevetsky, J. B. Bowles, Fourier analysis of numerical approximations of hyperbolic equations, SIAM Studies in Applied Mechanics, Philadelphia, 1982.

[48] T. Schonfeld, M. Rudgyard, Steady and unsteady flows simulations using the hybrid flow solver AVBP, AIAA Journal 37 (11) (1999) 1378-1385.

[49] AVBP, http://cerfacs.fr/avbp7x/.

[50] F. Nicoud, F. Ducros, Subgrid-scale stress modelling based on the square of the velocity gradient, Flow, Turb. and Combustion 62 (3) (1999) 183200 .

[51] P. I. Crumpton, J. A. Mackenzie, K. W. Morton, Cell vertex algorithms for the compressible Navier-Stokes equations, J. Comput. Phys. 109 (1993) 1-15.

[52] N. Lamarque, Schémas numériques et conditions limites pour la simulation aux grandes échelles de la combustion diphasique dans les foyers d'hélicoptère, Phd thesis, INP Toulouse (2007).

[53] M. Rudgyard, Cell vertex methods for steady inviscid flow, in: V. K. I. for Fluid Dynamics (Ed.), Lectures Series 1993-04, Vol. 1993-04, Von Karman Institute for Fluid Dynamics, 1993. 
[54] G. Puigt, V. Auffray, J.-D. Müller, Discretisation of diffusive fluxes on hybrid grids, J. Comput. Phys. 229 (2010) 1425-1447.

[55] V. Auffray, Etude comparative de schemas numeriques pour la modelisation de phenomenes diffusifs sur maillages multielements, Ph.D. thesis, Institut National Polytechnique de Toulouse (2007).

[56] P. D. Lax, B. Wendroff, Systems of conservation laws, Commun. Pure Appl. Math. 13 (1960) 217-237.

[57] P. D. Lax, B. Wendroff, Difference schemes for hyperbolic equations with high order of accuracy, Commun. Pure Appl. Math. 17 (1964) 381-398.

[58] L. Quartapelle, V. Selmin, High-order Taylor-Galerkin methods for nonlinear multidimensional problems., in: Finite Elements in Fluids, 1993, pp. $1374-1384$.

[59] J. Donea, A. Huerta, Finite Element Methods for Flow Problems, John Wiley \& Sons Inc, New York, 2003.

[60] O. Colin, M. Rudgyard, Development of high-order Taylor-Galerkin schemes for unsteady calculations, J. Comput. Phys. 162 (2) (2000) $338-371$.

[61] V. Moureau, G. Lartigue, Y. Sommerer, C. Angelberger, O. Colin, T. Poinsot, Numerical methods for unsteady compressible multicomponent reacting flows on fixed and moving grids, J. Comput. Phys. 202 (2) (2005) 710-736.

[62] F. Duchaine, S. Jauré, D. Poitou, E. Quémerais, G. Staffelbach, T. Morel, L. Gicquel, Analysis of high performance conjugate heat transfer with the openpalm coupler, Comp. Sci. Disc. 8 (1) (2015) 1500315021.

[63] Z. J. Wang, K. Fidkowski, R. Abgrall, F. Bassi, D. Caraeni, A. Cary, H. Deconinck, R. Hartmann, K. Hillewaert, H. T. Huynh, N. Kroll, G. May, P. Persson, B. van Leer, M. Visbal, High-order CFD methods: current status and perspective, Int. J. Numer. Meth. Fluids 72 (2013) 811-845. 
[64] T. Poinsot, S. Lele, Boundary conditions for direct simulations of compressible viscous flows, J. Comput. Phys. 101 (1) (1992) 104-129. doi:10.1016/0021-9991(92)90046-2.

[65] V. Granet, O. Vermorel, T. Leonard, L. Gicquel, , T. Poinsot, Comparison of nonreflecting outlet boundary conditions for compressible solvers on unstructured grids, AIAA Journal 48 (10) (2010) 2348-2364.

[66] T. Colonius, S. Lele, P. Moin, The free compressible vortex, J. Fluid Mech. 230 (1991) 45-73.

[67] S. W. Rienstra, A. Hirschberg, An introduction to acoustics, Eindhoven University of Technology, 2003.

[68] L. S. G. Kovasznay, Hot-wire investigation of the wake behind cylinders at low reynolds numbers, Proc. R. Soc. Lond. A, Mathematical and Physical Sciences 198 (1949) 174-190.

[69] C. H. K. Williamson, Defining a universal and continuous strouhalreynolds number relationship for the laminar vortex shedding of a circular cylinder, Phys. Fluids 31 (1988) 2742-2744.

[70] H. Persillon, M. Braza, Physical analysis of the transition to turbulence in the wake of a circular cylinder by three-dimensional Navier-Stokes simulation, J. Fluid Mech. 365 (1998) 23-88.

[71] A. Kravchenko, P. Moin, Numerical studies of flow over a circular cylinder at $\operatorname{Re}_{\mathrm{D}}=3900$, Phys. Fluids 12 (2) (2000) 403-417.

[72] A. W. Cook, W. H. Cabot, Hyperviscosity for shock-turbulence interactions, J. Comput. Phys. 203 (2005) 379-385. 
Click here to download LaTeX Source Files: JCP_JLaborderie_revised_version_archive.tar.gz 